\title{
OPTIMAL CONVERGENCE RESULTS FOR THE BREZZI-PITKÄRANTA APPROXIMATION OF THE STOKES PROBLEM: EXTERIOR DOMAINS
}

\author{
SERGUEI A. NAZAROV \\ Institute of Mechanical Engineering Problems \\ V.O. Bol'shoy Pr. 61, 199178 St. Petersburg, Russia \\ E-mail:serna@snark.ipme.ru \\ MARIA SPECOVIUS-NEUGEBAUER \\ Fachbereich 17, Mathematik/Informatik, Universität Kassel \\ D-34109 Kassel, Germany \\ E-mail: specovi@mathematik.uni-kassel.de
}

\begin{abstract}
This paper deals with a strongly elliptic perturbation for the Stokes equation in exterior three-dimensional domains $\Omega$ with smooth boundary. The continuity equation is substituted by the equation $-\varepsilon^{2} \Delta p+\operatorname{div} u=0$, and a Neumann boundary condition for the pressure is added. Using parameter dependent Sobolev norms, for bounded domains and for sufficiently smooth data we prove $H^{5 / 2-\delta}$ convergence for the velocity part and $H^{3 / 2-\delta}$ convergence for the pressure to the solution of the Stokes problem, with $\delta$ arbitrarily close to 0 . For an exterior domain the asymptotic behavior at infinity of the solutions to both problems has also to be taken into account. Although the usual Kondratiev theory cannot be applied to the perturbed problem, it is shown that the asymptotics of the solutions to the exterior Stokes problem and the solution to the perturbed problem coincide completely. For sufficiently smooth data an appropriate decay leads to the convergence of all main asymptotic terms as well as convergence in $H_{l o c}^{5 / 2-\delta}$ and $H_{l o c}^{3 / 2-\delta}$, respectively, of the remainder to the corresponding parts of the Stokes solution.
\end{abstract}

1. Introduction. We consider the following elliptic boundary value problems which appear in the context of viscous flow problems. In the first one we look for a solution $u^{\varepsilon}=\left(v_{1}^{\varepsilon}, v_{2}^{\varepsilon}, v_{3}^{\varepsilon}, p^{\varepsilon}\right)$ to

2000 Mathematics Subject Classification: Primary 76D07; Secondary 35Q30.

Key words and phrases: exterior Stokes problem, pressure stabilization method, singular perturbation.

The paper is in final form and no version of it will be published elsewhere. 


$$
\begin{array}{ll}
S_{\varepsilon} u=\left(\begin{array}{c}
-\Delta v+\nabla p \\
-\varepsilon^{2} \Delta p+\operatorname{div} v
\end{array}\right)=\left(\begin{array}{l}
f^{\prime} \\
f_{4}
\end{array}\right) \quad \text { in } \Omega, \\
B_{\varepsilon} u=\left(v, \partial_{n} p\right)=g & \text { on } \partial \Omega,
\end{array}
$$

where $f=\left(f_{1}, \ldots, f_{4}\right)=\left(f^{\prime}, f_{4}\right)$ is a given vector field on a three-dimensional domain $\Omega$ with a compact complement, $g=\left(g_{1}, \ldots, g_{4}\right)=\left(g^{\prime}, g_{4}\right)$ is a prescribed field on the boundary $\partial \Omega$, and $\varepsilon \in(0,1]$ is a small parameter. The system $\left(\mathrm{S}_{\varepsilon}\right)$ is strongly elliptic and can be accounted as a singular perturbation of the Stokes system:

$$
\begin{array}{ll}
S_{0} u=\left(\begin{array}{c}
-\Delta v+\nabla p \\
\operatorname{div} v
\end{array}\right)=\left(\begin{array}{l}
f^{\prime} \\
f_{4}
\end{array}\right) & \text { in } \Omega, \\
B_{0} u=v=g^{\prime} & \text { on } \partial \Omega .
\end{array}
$$

The Stokes problem - which is elliptic in the general sense of Agmon-Douglis-Nirenberg only-appears if we formally set $\varepsilon=0$ in $\left(\mathrm{S}_{\varepsilon}\right)$ and cancel the last boundary condition. We are interested what happens if $\varepsilon \searrow 0$, and, for $g_{4}=0$, to compare $u^{\varepsilon}$ and $u^{0}$. Perturbations of type $\left(\mathrm{S}_{\varepsilon}\right)$, usually with $f_{4}=0$ and homogeneous boundary conditions, appeared in different contexts, in showing existence of weak solutions for fluid models with shear dependent viscosities [4] as well as in numerical schemes for the Navier-Stokes equations, namely in the so-called pressure-stabilization methods, which were introduced by Brezzi and Pitkäranta in [2] (see also [11, 17], e.g.). In all these papers the problems were considered on bounded domains, then energy methods lead to the following estimate (see [17], e.g.), $f_{4}=0, g=0$ provided:

$$
\left\|v^{\varepsilon}-v^{0} ; H^{1}(\Omega)\right\|+\left\|p^{\varepsilon}-p^{0} ; L^{2}(\Omega)\right\| \leq C \varepsilon\left\|f^{\prime} ; L^{2}(\Omega)\right\| .
$$

In [10] asymptotically precise estimates with $\varepsilon \searrow 0$ were derived in the case of bounded smoothly surrounded domains. If the data are smooth enough and $g_{4}=0$ in $\left(\mathrm{S}_{\varepsilon}\right)$, then the velocity part $v^{\varepsilon}$ converges to $v^{0}$ in $H^{5 / 2-\delta}$ while the pressure converges in $H^{3 / 2-\delta}$ and $\delta>0$ can be arbitrarily small. Moreover, the convergence rate is increased, in the energy norms up to $O\left(\varepsilon^{3 / 2}\right)$. The results are sharp, which was proved by the construction of boundary layers.

In this paper we deal with exterior domains. For solutions to $\left(S_{0}\right)$ and $\left(S_{\varepsilon}\right)$ in unbounded domains, not only regularity properties, but also the asymptotic behavior as $r \rightarrow \infty$ is of importance. For the Stokes problem $\left(\mathrm{S}_{0}\right)$ there exists an exhaustive literature (see, e.g. [12] and the papers quoted there) while for the perturbed problem even the construction of a weak solution is not obvious. The theory of elliptic problems in Kondratiev spaces can be applied to the Stokes system and leads to explicit formulae for the asymptotic behavior of the solution $u^{0}$ in terms of the fundamental solution and their derivatives provided the right hand side $f$ decays quickly enough. However, this theory can not be applied to $u^{\varepsilon}$ since the problem $\left(\mathrm{S}_{\varepsilon}\right)$ is not admissible at infinity. Even the construction of weak solutions is not quite obvious. The appropriate function spaces for the problem $\left(\mathrm{S}_{\varepsilon}\right)$ are step weighted spaces, where the lowest derivatives can be multiplied with weights of Kondratiev type while the higher order derivatives are all supplied with the same weights. Results on the Stokes problem in step weighted spaces can be used to derive results on the asymptotics of the solutions of $\left(\mathrm{S}_{\varepsilon}\right)$ as well. Surprisingly 
enough it turns out that the asymptotic expansions at infinity of $u^{\varepsilon}$ and $u^{0}$ coincide completely. Based on these results it is possible to consider $u^{\varepsilon}$ as well as $u^{0}$ as elements of the same function space with separated asymptotic terms and derive asymptotically precise estimates for the differences of the asymptotic terms as well as for the remainders as $\varepsilon \searrow 0$.

The plan of the paper is the following. In Section 2 we refer to general notations and recall the results on bounded domains. In Section 3 we recall the notations for Kondratiev spaces and the results for the exterior Stokes problem and adopt them to weighted Sobolev spaces with noninteger differentiability index. In Section 4 we introduce step weighted spaces and investigate the solutions to $\left(\mathrm{S}_{0}\right)$ in these function spaces. In Section 5 we derive a priori estimates in step weighted spaces, the first main result is the theorem on the asymptotic behavior of the solutions $u^{\varepsilon}$ (Theorem 5.2). The last two sections contain the other main results of this paper: Under the condition that the weight indices fulfil the same restrictions as in the corresponding result for the Stokes problem, the problem $\left(\mathrm{S}_{\varepsilon}\right)$ is uniquely solvable in step weighted spaces (Theorem 6.2). Together with the results on the asymptotic expansions this implies: If the right hand side $f$ decays quickly enough, both problems possess a uniquely determined solution vanishing at infinity, $u^{\varepsilon}$ and $u^{\varepsilon}$ can be decomposed into a finite sum in terms of derivatives of the fundamental solution to the Stokes system and a quicker decaying remainder. The last result (Theorem 7.2) gives optimal error estimates for the coefficients of the separated asymptotic terms, while the remainder can be estimated in weighted Sobolev norms.

2. Some general notations, the results in bounded domains. For $G \subset \mathbb{R}^{3}$, the closure of $G$ is denoted by $\bar{G}$, the boundary by $\partial G$, and for $x \in \partial G$ the exterior unit normal vector by $n(x)$, if it exists. The modulus function in $\mathbb{R}^{3}$ is named $r$, i.e. $r(x)=\left(\sum x_{i}^{2}\right)^{1 / 2}$. For any $t \in \mathbb{R}$ we call $[t]$ the integer part of $t$, i.e. $[t]=\max \{j \in \mathbb{Z}: j \leq t\}$, while the number $t_{+}=(t+|t|) / 2$ means the positive part of $t$.

For partial derivatives we use the common multi-index terminology: $\partial^{\alpha}=\frac{\partial^{\alpha_{1}}}{\partial x_{1}} \frac{\partial^{\alpha_{2}}}{\partial x_{2}} \frac{\partial^{\alpha_{3}}}{\partial x_{3}}$, with $\alpha \in \mathbb{N}_{0}^{3}$. If $k \in \mathbb{N}$, then $\nabla^{k}$ indicates the collection of all partial derivatives of order $k$.

Depending on our problems, we need different kinds of function spaces, most of them are introduced in the context. For any function space $X$, we indicate the norm in $X$ by $\|\cdot ; X\|$. We recall the notations for some standard spaces. $C_{0}^{\infty}(G), C_{0}^{\infty}(\bar{G})$ are the spaces of all smooth functions with compact support in $G$ and $\bar{G}$, respectively. For $l \in \mathbb{N}$, the Sobolev space $H^{l}(G),\left(G \subset \mathbb{R}^{3}\right.$ open $)$ consists of all $\varphi \in L^{2}(G)$ with distributional derivatives $\partial^{\alpha} \varphi \in L^{2}(G),|\alpha| \leq l$, supplied with the usual norm. If $s>0, s \notin \mathbb{N}$, then the Sobolev-Slobodetskii space $H^{s}(G)=\left\{\varphi \in H^{[s]}(G):\left\|\varphi ; H^{s}(G)\right\|<\infty\right\}$, where

$$
\left\|\varphi ; H^{s}(G)\right\|^{2}=\left\|v ; H^{[s]}(G)\right\|^{2}+\sum_{|\alpha|=[s]} \int_{G} \int_{G} \frac{\left|\partial^{\alpha} \varphi(x)-\partial^{\alpha} \varphi(\xi)\right|^{2}}{|x-\xi|^{n+2(s-[s])}} d \xi d x .
$$

Furthermore, $H^{-s}(G)$ is the dual space of $\stackrel{\circ}{H}^{s}(G)$, the supscript $\circ$ indicates the closure of $C_{0}^{\infty}(G)$ in $H^{s}(G)$. For $\varphi \in H^{-s}(G)$ we have the non-unique representation

$$
\varphi=\sum_{|\alpha| \leq[s]+1} \partial^{\alpha} \varphi^{\alpha}, \quad \varphi^{\alpha} \in H^{[s]+1-|\alpha|}(G),
$$


where with the infimum taken over all possible representations, the norm in $H^{-s}(G)$ is equivalent to

$$
\left\|\varphi ; H^{-s}(G)\right\|=\inf \left\{\left(\sum_{|\alpha| \leq[s]+1}\left\|\varphi^{\alpha} ; H^{[s]+1-|\alpha|}(G)\right\|\right)^{1 / 2}\right\} .
$$

If $\partial G$ is compact and sufficiently smooth, i.e. of class $C^{[s]+2}$, for example, then $H^{s}(\partial G)$ is defined, by using local coordinates and a partition of unity on $\partial G$, via the definition of $H^{s}\left(\mathbb{R}^{2}\right)$, while $H^{-s}(\partial G)$ is the dual space of $H^{s}(\partial G)$ (see [7] for details).

Now let $G \subset \mathbb{R}^{3}$ be a bounded domain with $\partial G \in C^{l+2}, l \in \mathbb{N}_{0}$. To obtain asymptotically precise estimates for solutions $u^{\varepsilon}$ to problem $\left(\mathrm{S}_{\varepsilon}\right)$, we supply the spaces $H^{l}(G)$ with equivalent norms, where the small parameter $\varepsilon \in(0,1]$ is included (see $[8,10])$. For $\varkappa \leq l$ and $\varphi \in H^{l}(G)$ we set

$$
\left\|\varphi ; H_{\varkappa}^{l}(G ; \varepsilon)\right\|^{2}=\left\|\varphi ; H^{\varkappa}(G)\right\|^{2}+\sum_{k=0}^{l} \varepsilon^{2(k-\varkappa)+\left\|\nabla \varphi ; L^{2}(G)\right\|^{2} .}
$$

Then for any differential operator $\partial^{\alpha}$ with $|\alpha| \leq l$, and $k$ with $\varkappa+k \leq l$, we have

$$
\begin{aligned}
\left\|\partial^{\alpha} \varphi ; H_{\varkappa-|\alpha|}^{l-|\alpha|}(G ; \varepsilon)\right\| & \leq C\left\|\varphi ; H_{\varkappa}^{l}(G ; \varepsilon)\right\|, \\
\left\|\varepsilon^{k} \partial^{\alpha} \varphi ; H_{\varkappa-|\alpha|+k}^{l-|\alpha|}(G ; \varepsilon)\right\| & \leq C\left\|\varphi ; H_{\varkappa}^{l}(G ; \varepsilon)\right\| .
\end{aligned}
$$

We process the trace spaces $H^{l-1 / 2}(\partial G)$ in the following way. We define

$$
\left\|v ; \mathcal{H}_{\varkappa-1 / 2}^{l-1 / 2}(\partial G ; \varepsilon)\right\|=\left\{\begin{array}{r}
\left(\left\|v ; H^{\varkappa-1 / 2}(\partial G)\right\|^{2}+\varepsilon^{2(l-\varkappa)}\left\|v ; H^{l-1 / 2}(\partial G)\right\|^{2}\right)^{1 / 2} \\
\text { for } \varkappa>1 / 2 ; \\
\varepsilon^{-\varkappa+1 / 2}\left(\left\|v ; L^{2}(\partial G)\right\|^{2}+\varepsilon^{2 l-1}\left\|v ; H^{l-1 / 2}(\partial G)\right\|^{2}\right)^{1 / 2} \\
\text { for } \varkappa<1 / 2 .
\end{array}\right.
$$

Then the norms of the trace operators $\partial_{n}^{h}: H_{\varkappa}^{l}(G ; \varepsilon) \rightarrow H_{\varkappa-h-1 / 2}^{l-h-1 / 2}(\partial G ; \varepsilon)$, where $h=0$ and $h=1$, can be bounded independent of $\varepsilon \in(0,1]$, the converse result on prolongations is also true.

Theorem 2.1 ([10]). Let $G \subset \mathbb{R}^{3}$ be a bounded domain with $\partial G$ of class $C^{l+2}, l \in \mathbb{N}$ and let $\varkappa \in[0,3 / 2), \varkappa \leq l-1$. Then for any $f \in H^{l-1}(G)^{4}$ and $g=\left(g^{\prime}, g_{4}\right) \in H^{l+1 / 2}(G)^{3} \times$ $H^{l}(G)$ subject to the compatibility condition

$$
\int_{G} f_{4}-\int_{\partial G} g^{\prime} \cdot n d o+\varepsilon^{2} \int_{\partial G} g_{4} d o=0,
$$

there exists a solution $u^{\varepsilon}=\left(v^{\varepsilon}, p^{\varepsilon}\right) \in H^{l+1}(G)$ to problem $\left(\mathrm{S}_{\varepsilon}\right)$, which is unique under the orthogonality condition $\int_{G} p^{\varepsilon}=0$. This solution satisfies the estimate

$$
\begin{aligned}
& \left\|v^{\varepsilon} ; H_{\varkappa+1}^{l+1}(G ; \varepsilon)\right\|+\left\|p^{\varepsilon} ; H_{\varkappa, \perp}^{l+1}(G ; \varepsilon)\right\| \\
& \leq C\left(\left\|f^{\prime} ; H_{\varkappa-1}^{l-1}(G ; \varepsilon)\right\|+\left\|f_{4} ; H_{\varkappa}^{l-1}(G ; \varepsilon)\right\|\right. \\
& \left.\quad+\left\|g^{\prime} ; H_{\varkappa+1 / 2}^{l+1 / 2}(\partial G ; \varepsilon)\right\|+\left\|g_{4} ; H_{\varkappa-3 / 2}^{l-3 / 2}(G ; \varepsilon)\right\|\right),
\end{aligned}
$$

where $C$ is a constant depending on $\varkappa, \partial G$ and $l$, but neither on $\varepsilon \in(0,1]$ nor on the data $(f, g)$. The subscript $\perp$ indicates the subspace of mean-value-free functions. 
REMARK 2.2. In [10] even $\varkappa \leq l$ was admissible, this uses $H_{\varkappa}^{m}(G ; \varepsilon)$-norms, where $\varkappa>m$. Theorem 2.1 is based on an interior estimate of the following type: Let $G^{\prime}$ be a strictly interior subdomain of $G$, i.e. $\bar{G}^{\prime} \subset G$, then

$$
\begin{aligned}
& \left\|v^{\varepsilon} ; H_{\varkappa+1}^{l+1}\left(G^{\prime} ; \varepsilon\right)\right\|+\left\|p^{\varepsilon} ; H_{\varkappa}^{l+1}\left(G^{\prime} ; \varepsilon\right)\right\| \\
& \quad \leq C\left(\left\|f^{\prime} ; H_{\varkappa-1}^{l-1}(G ; \varepsilon)\right\|+\left\|f_{4} ; H_{\varkappa}^{l-1}(G ; \varepsilon)\right\|+\left\|u^{\varepsilon} ; L^{2}(G)\right\|\right),
\end{aligned}
$$

where $C$ does not depend on $\varepsilon$.

\section{The exterior Stokes problem in Kondratiev spaces}

3.1. Kondratiev spaces and the scaling trick. For the exterior Stokes problem, the appropriate function spaces are the Kondratiev spaces $V_{\beta}^{s}(\Omega)$. We require that $\Omega \subset \mathbb{R}^{3}$ is a domain with a compact complement $\mathbb{R}^{3} \backslash \Omega$ and with boundary $\partial \Omega$ at least of class $C^{2}$. For simplicity, we assume that $\mathbb{R}^{3} \backslash \bar{\Omega}$ is non void and contains the point 0 . If $s \in \mathbb{N}_{0}=\{0,1,2, \ldots\}$, and $\beta \in \mathbb{R}$, the Kondratiev space $V_{\beta}^{s}(\Omega)$ is defined as the closure of $C_{0}^{\infty}(\bar{\Omega})$ with respect to the norm $\left\|\cdot ; V_{\beta}^{s}(\Omega)\right\|$, where

$$
\left\|\varphi ; V_{\beta}^{s}(\Omega)\right\|^{2}=\sum_{|\alpha| \leq s}\left\|r^{\beta-s+|\alpha|} \partial^{\alpha} \varphi ; L^{2}(\Omega)\right\|^{2} .
$$

If $s>0$ is non-integer, then (3.1) is changed for

$$
\left\|\varphi ; V_{\beta}^{s}(\Omega)\right\|^{2}=\left\|\varphi ; V_{\beta-s+[s]}^{[s]}(\Omega)\right\|^{2}+\sum_{|\alpha|=[s]} \int_{\Omega}|x|^{2 \beta} \int_{\ldots} \frac{\left|\partial^{\alpha} \varphi(x)-\partial^{\alpha} \varphi(y)\right|^{2}}{|x-y|^{3+2(s-[s])}} d y d x,
$$

where the $\operatorname{dots} \ldots$ stand for $\left\{y \in \Omega:|x-y|<2^{-1}|x|\right\}$. Finally, in the case $s<0$, the inclusion $\varphi \in V_{\beta}^{s}(\Omega)$ means that $\varphi$ is a distribution which can be represented in the form

$$
\begin{aligned}
\varphi & =\sum_{|\alpha| \leq-[s]} \partial^{\alpha} \varphi^{\alpha}, \text { with } \varphi^{\alpha} \in V_{\beta-s-|\alpha|+(s+|\alpha|)_{+}}^{(s+|\alpha|)_{+}}(\Omega), \\
\left\|\varphi ; V_{\beta}^{s}(\Omega)\right\| & =\inf \left(\sum_{|\alpha| \leq-[s]}\left\|\varphi^{\alpha} ; V_{\beta-s-|\alpha|+(s+|\alpha|)_{+}}^{(s+|\alpha|)_{+}}(\Omega)\right\|^{2}\right)^{1 / 2},
\end{aligned}
$$

where the infimum is taken over all representations (3.2). Note that always $V_{\beta}^{s}(\Omega) \subset$ $H_{l o c}^{s}(\bar{\Omega})$ is valid $^{1}$. Since $\partial \Omega$ is compact, for $s>1 / 2$, the trace operator $\Gamma: V_{\beta}^{s}(\Omega) \rightarrow$ $H^{s-1 / 2}(\partial \Omega)$ with $\Gamma \varphi=\left.\varphi\right|_{\partial \Omega}$ for smooth functions, is well defined and continuous, with a norm bounded independent of $\beta$. It is clear, that any differential operator $\partial^{\alpha}$ acts linearly and continuously from $V_{\beta}^{s}(\Omega)$ to $V_{\beta}^{s-|\alpha|}(\Omega)$.

One of the main arguments in the following proofs is the scaling trick. It is used to reduce estimates for the solutions $u^{\varepsilon}, u^{0}$ in function spaces on $\Omega$ to estimates to the corresponding problems in bounded domains. The idea is to write $\Omega=\bigcup_{k=k_{0}}^{\infty} G_{k}$, where all $G_{k} \subset \Omega$ are bounded domains such that the modulus function $r$ is equivalent to a constant $c_{k}$ on $G_{k}$, and then use a suitable coordinate transformation in order to transform $G_{k}$ into a fixed domain $\Xi$ for all $k>k_{0}$. To realize this, we fix $j \in\{1,2\}$ and

\footnotetext{
${ }^{1}$ As a matter of fact, $V_{\beta}^{s}(\Omega)=\left\{\varphi \in H_{l o c}^{s}(\bar{\Omega}):\left\|\varphi ; V_{\beta}^{s}(\Omega)\right\|<\infty\right\}$.
} 
$k_{0} \in \mathbb{N}$ large enough such that $\partial \Omega \subset\left\{x: r<2^{k_{0}-j}\right\}$. We put

$$
G_{k_{0}}=\Omega \cap\left\{x: r<2^{k_{0}+j}\right\} \text { and } G_{k}=\left\{x: 2^{k-j}<r<2^{k+j}\right\} \text { for } k>k_{0} .
$$

Then $\Omega=\bigcup_{k \geq k_{0}} G_{k}$ and for $k>k_{0}$, the transformation $x \mapsto \xi:=2^{-k} x$ maps $G_{k}$ onto $\Xi=\left\{\xi: 2^{-j}<|\xi|<2^{j}\right\}$. Let $\varphi \in V_{\gamma}^{0}(\Omega)$ for some $\gamma \in \mathbb{R}$. Since $r \sim 2^{k}$ in $G_{k}$, independent of $k$, we have

$$
\left\|r^{\gamma} \varphi ; L^{2}(\Omega)\right\|^{2} \sim \sum_{k \geq k_{0}} 2^{2 \gamma}\left\|\varphi ; L^{2}\left(G_{k}\right)\right\|^{2}
$$

where the equivalence constants depend on $\gamma$ and $j$ only. For $\xi \in \Xi, \varphi \in L_{l o c}^{2}(\bar{\Omega})$, we put

$$
\widehat{\varphi}_{k}(\xi)=\varphi\left(2^{k} \xi\right) .
$$

With these notations, we collect the estimates of the rescaling method in the following lemma.

LEMmA 3.1. Let $s \in \mathbb{R}, \beta \in \mathbb{R}, h \in \mathbb{N}_{0}$ and $\varphi \in L_{\text {loc }}^{2}(\bar{\Omega})$, then the following equivalences hold, whenever the left-hand side is finite,

$$
\begin{aligned}
\left\|r^{\beta} \nabla_{x}^{h} \varphi ; L^{2}(\Omega)\right\|^{2} & \sim\left\|\nabla_{x}^{h} \varphi ; L^{2}\left(G_{k_{0}}\right)\right\|^{2}+\sum_{k>k_{0}}\left(2^{k}\right)^{2 \beta-2 h+3}\left\|\nabla_{\xi}^{h} \widehat{\varphi}_{k} ; L^{2}(\Xi)\right\|^{2}, \\
\left\|\varphi ; V_{\beta}^{s}(\Omega)\right\|^{2} & \sim\left\|\varphi ; H^{s}\left(G_{k_{0}}\right)\right\|^{2}+\sum_{k>k_{0}}\left(2^{k}\right)^{2 \beta-2 s+3}\left\|\widehat{\varphi}_{k} ; H^{s}(\Xi)\right\|^{2},
\end{aligned}
$$

here all equivalence constants depend on $s, \beta, j$ and $h$.

Proof. Relation (3.5) follows from (3.3) by substituting in $\left\|\nabla^{h} \varphi ; L^{2}\left(G_{k}\right)\right\|$ the expressions

$$
\nabla_{x}^{h} \varphi=2^{-k h} \nabla_{\xi}^{h} \widehat{\varphi}_{k}, \quad d x=2^{3 k} d \xi
$$

Then for $s \in \mathbb{N}$, (3.6) is obtained from (3.5) by replacing the weight exponent $\beta$ in (3.5) by the exponents $\beta-s+|\alpha|$.

For non integer $s>0$, we have to observe that due to $j \geq 1$, the intersection $G_{k} \cap G_{k+1}$ contains at least the annulus $\left\{x: 2^{k}<|x|<2^{k+1}\right\}$. Thus,

$$
\begin{aligned}
& \int_{\Omega} r^{\beta} \int_{|x-y| \leq \frac{1}{2}|x|} \frac{\left|\nabla^{[s]} \varphi(x)-\nabla^{[s]} \varphi(y)\right|^{2}}{|x-y|^{3+2(s-[s])}} d y d x \\
& \quad \sim \sum_{k \geq k_{0}} 2^{2 k \beta} \int_{G_{k}} \int_{\left\{y \in G_{k}:|x-y| \leq|x| / 2\right\}} \frac{\left|\nabla^{[s]} \varphi(x)-\nabla^{[s]} \varphi(y)\right|^{2}}{|x-y|^{3+2(s-[s])}} d y d x .
\end{aligned}
$$

Using $x=2^{k} \xi, y=2^{k} \eta$, and the previous considerations, lead to (3.6) in this case, too.

It remains to consider the case $s<0$. We have

$$
\left\|\varphi ; V_{\beta}^{s}(\Omega)\right\|^{2}=\inf \left\{\sum_{|\alpha| \leq[-s]}\left\|\varphi^{\alpha} ; V_{\beta-s-|\alpha|}^{0}(\Omega)\right\|+\sum_{|\alpha|=-[s]}\left\|\varphi^{\alpha} ; V_{\beta}^{s-[s]}(\Omega)\right\|\right\},
$$

where the infimum is taken over all possible representations $(3.2)$ (observe $-[s]=[-s]+1$, if $s \notin \mathbb{Z})$. By $(3.7)$, we obtain for $\xi \in \Xi$ :

$$
\widehat{\varphi}_{k}(\xi)=\sum_{\mid \alpha \leq-[s]} 2^{-k|\alpha|} \partial_{\xi}^{\alpha} \widehat{\varphi}_{k}^{\alpha}(\xi)
$$


Since we are free to vary the representations locally, the right-hand sides of (3.9) run through all possible representations of $\widehat{\varphi}_{k}(\xi)$ on $\Xi$, and hence

$$
\left\|\widehat{\varphi}_{k} ; H^{s}(\Xi)\right\|^{2} \sim \inf \left\{\sum_{|\alpha| \leq-[s]}\left\|2^{-k|\alpha|} \widehat{\varphi}_{k}^{\alpha} ; L^{2}(\Xi)\right\|^{2}+\sum_{|\alpha|=-[s]}\left\|2^{k[s]} \widehat{\varphi}_{k}^{\alpha} ; H^{s-[s]}(\Xi)\right\|^{2}\right\} .
$$

By (3.5) and (3.6) for $s>0$, the right-hand side of (3.8) is equivalent to

$$
\begin{aligned}
\inf \left\{\sum_{k \geq k_{0}} 2^{2 k(\beta-s)}\left(\sum_{|\alpha|<-[s]}\left\|2^{-k|\alpha|} \varphi^{\alpha} ; L^{2}\left(G_{k}\right)\right\|^{2}+\sum_{|\alpha|=-[s]}\left\|2^{k[s]} \varphi^{\alpha} ; H^{s-[s]}\left(G_{k}\right)\right\|^{2}\right)\right\} \\
=\inf \left\{\text { terms on } G_{k_{0}}+\right. \\
\left.\quad \sum_{k>k_{0}} 2^{2 k(\beta-s+3 / 2)}\left(\sum_{|\alpha|<-[s]}\left\|2^{-k|\alpha|} \widehat{\varphi}_{k}^{\alpha} ; L^{2}(\Xi)\right\|^{2}+\sum_{|\alpha|=-[s]}\left\|2^{k[s]} \widehat{\varphi}_{k}^{\alpha} ; H^{s-[s]}(\Xi)\right\|^{2}\right)\right\},
\end{aligned}
$$

the infimum is always taken over all representations $(3.2)$. Since $\sum_{k} \inf \ldots \leq \inf \sum_{k} \ldots$, this leads to

$$
\left\|\varphi ; H^{s}\left(G_{k_{0}}\right)\right\|^{2}+\sum_{k>k_{0}} 2^{2 k(\beta-s+3 / 2)}\left\|\widehat{\varphi}_{k} ; H^{s}(\Xi)\right\|^{2} \leq C(s, \beta, j, \partial \Omega)\left\|\varphi ; V_{\beta}^{s}(\Omega)\right\|^{2} .
$$

To see the reverse inequality, we decompose $\varphi=\sum_{k \geq k_{0}} \zeta_{k} \varphi$, where $\left\{\zeta_{k}\right\}_{k}$ is a partition of unity subordinated to the covering $\left\{G_{k}\right\}$, with $\partial^{\alpha} \bar{\zeta}_{k}$ uniformly bounded independent of $k$ and $|\alpha| \leq-[s]$, and find

$$
\left\|\varphi ; V_{\beta}^{s}(\Omega)\right\|^{2} \leq C \sum_{k \geq k_{0}}\left\|\varphi ; V_{\beta}^{s}\left(G_{k}\right)\right\|^{2}
$$

Here for $k>k_{0}$,

$$
\begin{aligned}
\left\|\varphi ; V_{\beta}^{s}\left(G_{k}\right)\right\|= & 2^{k(\beta-s)} \inf \left\{\sum_{|\alpha|<-[s]} 2^{-k|\alpha|}\left\|\varphi_{(k)}^{\alpha} ; L^{2}\left(G_{k}\right)\right\|\right. \\
& \left.+\sum_{|\alpha|=-[s]}\left\|2^{k[s]} \varphi_{(k)}^{\alpha} ; H^{s-[s]}\left(G_{k}\right)\right\|\right\},
\end{aligned}
$$

where now the infimum is taken over every representation

$$
\left.\varphi\right|_{G_{k}}=\sum_{|\alpha| \leq-[s]} \partial^{\alpha} \varphi_{(k)}^{\alpha}
$$

separately on each $G_{k}$. Using (3.7) again, we calculate from (3.10) for $k>k_{0}$ :

$$
\left\|\varphi ; V_{\beta}^{s}\left(G_{k}\right)\right\|=2^{k(\beta-s+3 / 2)}\left\|\widehat{\varphi}_{k} H^{s}(\Xi)\right\|,
$$

which finishes the proof.

3.2. The Stokes problem in Kondratiev spaces. Let $s \geq 0$, to the Stokes problem $\left(\mathrm{S}_{0}\right)$ we associate a natural domain for the solutions,

$$
\mathscr{D}_{\beta}^{s} V(\Omega)=V_{\beta}^{s+1}(\Omega)^{3} \times V_{\beta}^{s}(\Omega),
$$

a natural range for the set of data,

$$
\mathscr{R}_{\beta}^{s} V(\Omega)=V_{\beta}^{s-1}(\Omega)^{3} \times V^{s}(\Omega) \times H^{s+1 / 2}(\partial \Omega)^{3},
$$


and the linear continuous operator

$$
A_{\beta}^{s}: \mathscr{D}_{\beta}^{s} V(\Omega) \ni u=(v, p) \mapsto\left(S_{0} u, B_{0} u\right) \in \mathscr{R}_{\beta}^{s} V(\Omega) .
$$

To state the result on the Fredholm properties of $A_{\beta}^{s}$, we recall the notion of the power solution. For $\lambda \in \mathbb{C}$, a power solution of generalized degree $\lambda$ is a solution

$$
U(x)=(V(x), P(x))=\left(r^{\lambda} \mathcal{V}(\phi), r^{\lambda-1} \mathcal{P}(\phi)\right), \quad \text { with } \phi=r^{-1} x \in \mathbb{S}^{2},
$$

to the homogeneous Stokes system in the punctured space:

$$
-\Delta V+\nabla P=0, \quad \operatorname{div} V=0 \quad \text { in } \mathbb{R}^{3} \backslash\{0\} .
$$

It is known (see [9, Ch. 6], e.g.) that any such solution is either a polynomial or a linear combination of derivatives $\partial^{\alpha} \mathcal{E}^{j}$, where $\mathcal{E}^{j}, j=1, \ldots, 4$ are the columns of the fundamental solution matrix in $\mathbb{R}^{3}$. Since

$$
\begin{aligned}
\mathcal{E}_{i}^{j}(x) & =(8 \pi)^{-1}\left(\delta_{i j}|x|^{-1}+x_{i} x_{j}|x|^{-3}\right), 1 \leq i, j \leq 3, \\
\mathcal{E}_{j}^{4}(x) & =\mathcal{E}_{4}^{j}(x)=(4 \pi)^{-1} x_{j}|x|^{-3}, 1 \leq j \leq 3, \\
\mathcal{E}_{44}(x) & =\delta(x) \quad \text { (the Dirac measure), }
\end{aligned}
$$

we see that $\mathcal{E}^{1}, \mathcal{E}^{2}, \mathcal{E}^{3}$ are power solutions of generalized degree $\lambda=-1$, while $\mathcal{E}^{4}$ can be regarded as a power solution of generalized degree $\lambda=-2$. Moreover, it is clear that in $\mathbb{R}^{3} \backslash\{0\}$, power solutions of degree $\lambda$ exist if and only if $\lambda \in \mathbb{Z}$. We have the following result on existence, uniqueness and asymptotics of solutions to the exterior Dirichlet problem $\left(\mathrm{S}_{0}\right)$.

THEOREM 3.2. (i) Let $s \geq 1$ and $\left(f, g^{\prime}\right) \in \mathscr{R}_{\beta}^{s} V(\Omega)$ and $u \in H_{l o c}^{s+1}(\bar{\Omega})^{3} \times H_{l o c}^{s}(\bar{\Omega})$ be a solution to the Stokes problem $\left(\mathrm{S}_{0}\right)$ with $u \in V_{\beta-s-1}^{0}(\Omega)^{2} \times V_{\beta-s}^{0}(\Omega)$, then $u \in \mathscr{D}_{\beta}^{s} V(\Omega)$, and

$$
\left\|u ; \mathscr{D}_{\beta}^{s} V(\Omega)\right\| \leq C\left(\left\|\left(f, g^{\prime}\right) ; \mathscr{R}_{\beta}^{s} V(\Omega)\right\|+\left\|u ; V_{\beta-s-1}^{0}(\Omega)\right\|+\left\|p ; V_{\beta-s}^{0}(\Omega)\right\|\right) .
$$

For $s \in[0,1]$, it is sufficient to require $p \in H_{l o c}^{s} \cap V_{\beta-s-1}^{-1}(\Omega)$ and replace the corresponding norm in (3.15).

(ii) For any $s \geq 0$, the operator $A_{\beta}^{s}$ is Fredholm iff there exists no power solution of the form (6) with $\lambda=\beta-s-1 / 2$, i.e. iff

$$
\beta-s-1 / 2 \notin \mathbb{Z} \text {. }
$$

(iii) The operator $A_{\beta}^{s}$ is injective, iff $\beta-s \geq-1 / 2$, and surjective iff (3.16) and $\beta-s<$ $1 / 2$, thus $A_{\beta}^{s}$ is an isomorphism iff $|\beta-s|<1 / 2$.

(iv) Assume $\beta$ as well as $\gamma$ fulfil (3.16) and $\beta<\gamma<\beta+1$. Let $u=(v, p) \in \mathscr{D}_{\beta}^{s} V(\Omega)$ be given with $\left(f, g^{\prime}\right)=A_{\beta}^{s} u \in \mathscr{R}_{\gamma}^{s} V(\Omega)$. Then $u$ has the following asymptotic representation

$$
u=U+\tilde{u}, \text { where } U=\sum_{j=1}^{J} a_{j} U^{j}, \quad \tilde{u} \in \mathscr{D}_{\gamma}^{s} V(\Omega),
$$

together with the estimate

$$
\left\|\tilde{u} ; \mathscr{D}_{\gamma}^{s} V(\Omega)\right\|+\sum_{j=1}^{j}\left|a_{j}\right| \leq C\left(\left\|\left(f, g^{\prime}\right) ; \mathscr{R}_{\gamma}^{s} V(\Omega)\right\|+\left\|u ; V_{\beta-s-1}^{0}(\Omega)^{3} \times V_{\beta-s}^{0}(\Omega)\right\|\right) .
$$


Here $U$ is a power solution of generalized degree $\lambda$ which is an integer in the interval $I=(s-\gamma-1 / 2, s-\beta-1 / 2)$, the set $\left\{U^{1}, \ldots, U^{J}\right\}$ forms a basis for the corresponding vector space of power solutions. If $I \cap \mathbb{Z}=\emptyset$, then the $\sum \ldots$ in (3.17) and (3.18) is void, which implies $u \in \mathscr{D}_{\gamma}^{s} V(\Omega)$ then.

Proof. For an integer $s \in \mathbb{N}$ the assertions (i)-(iv) can be found e.g. in Chapter 6.4 of [9] and also in [15]. Combining the results of [14] with those of [3] and [15] they extend to $s=0$. For $s>0$, it is enough therefore to prove the a priori estimate

$$
\left\|u ; \mathscr{D}_{\beta}^{s} V(\Omega)\right\| \leq C\left(\left\|\left(S_{0} u, B_{0} u\right) ; \mathscr{R}_{\beta}^{s} V(\Omega)\right\|+\left\|u ; V_{\beta-s-1}^{0}(\Omega)^{3} \times V_{\beta-s-1}^{-1}(\Omega)\right\|\right) .
$$

Since $\left\|p ; V_{\beta-s-1}^{-1}(\Omega)\right\| \leq\left\|p ; V_{\beta-s}^{0}(\Omega)\right\|$, we have (3.15) then, too. We employ the method of increasing the local smoothness (see, e.g. [18]) together with Lemma 3.1.

Let $G \subset \mathbb{R}^{3}$ be a bounded domain with $\partial G$ of class $C^{[s]+2}$. Then the following a priori estimates hold:

$$
\begin{gathered}
\left\|v ; H^{s+1}(G)\right\|^{2}+\left\|p ; H^{s}(G)\right\|^{2} \leq C_{G}\left(\left\|S_{0} u ; H^{s-1}(G)^{3} \times H^{s}(G)\right\|^{2}\right. \\
\left.+\left\|v ; H^{s-1 / 2}(\partial G)\right\|+\left\|v ; L^{2}(G)\right\|^{2}+\left\|p ; H^{-1}(G)\right\|^{2}\right),
\end{gathered}
$$

for any $u=(v, p) \in H^{s+1}(G)^{3} \times H^{2}(G)$, furthermore the interior estimate

$$
\begin{aligned}
& \left\|v ; H^{s+1}\left(G^{\prime}\right)\right\|+\left\|p ; H^{s}\left(G^{\prime}\right)\right\| \\
& \quad \leq C\left(\left\|S_{0} u ; H^{s-1}(G)^{3} \times H^{s}(G)\right\|+\left\|v ; L^{2}(G)\right\|+\left\|p ; H^{-1}(G)\right\|\right),
\end{aligned}
$$

where $G^{\prime} \subset G$ is a strictly interior subdomain, i.e. $\bar{G}^{\prime} \subset G$ (see Theorem 10.1.1 in [18], Theorem 6.4 .8 in [5] for $s>1 / 2$, for $s \in[0,1 / 2)$ the inequalities (3.20) and (3.21) follow from the results of [3] and interpolation arguments). We define the set of subdomains $\left\{G_{k}^{\prime}\right\}_{k \geq k_{0}}$ as in Lemma 3.1, where we choose $j=1$, and $\left\{G_{k}\right\}_{k \geq k_{0}}$ with $G_{k} \supset G_{k}^{\prime}$ in the same way, but with $j=2$. Then for $k>k_{0}$, the transformation $x \mapsto \xi=2^{-k} x$ transforms $G_{k}^{\prime}, G_{k}$ into $\Xi^{\prime}$ and $\Xi$, with $\Xi^{\prime} \subset \Xi=\{1 / 4 \leq|\xi| \leq 4\}$. With $\hat{v}_{k}(\xi), \hat{p}_{k}(\xi)$ as in (3.4) we calculate by means of (3.7) that for $k>k_{0}$

$$
-\Delta_{\xi} \hat{v}_{k}+\nabla_{x}\left(2^{k} \hat{p}_{k}\right)=2^{2 k} \hat{f}_{k}^{\prime}, \quad \operatorname{div}_{\xi} \hat{v}_{k}=2^{k} \hat{f}_{4, k}
$$

on $\Xi$, where $\left(f^{\prime}, f_{4}\right)=S_{0} u$. We apply (3.20) on $G_{k_{0}}^{\prime}$ and (3.21) on $\Xi^{\prime}$ and $\Xi$, which leads to

$$
\begin{aligned}
& \left\|\hat{v}_{k} ; H^{s+1}\left(\Xi^{\prime}\right)\right\|^{2}+2^{2 k}\left\|\hat{p} ; H^{s}\left(\Xi^{\prime}\right)\right\|^{2} \\
& \leq C\left(2^{4 k}\left\|\hat{f}_{k}^{\prime} ; H^{s-1}(\Xi)\right\|^{2}+2^{2 k}\left\|\hat{f}_{4, k} ; H^{s}(\Xi)\right\|^{2}+\left\|\hat{v}_{k} ; L^{2}(\Xi)\right\|+2^{2 k}\left\|\hat{p}_{k} ; H^{-1}(\Xi)\right\|^{2}\right),
\end{aligned}
$$

where $C$ is independent of $k$. Multiplying these series of inequalities by $2^{2 k(\beta-s-1+3 / 2)}$ and then using (3.6) leads to (3.19), if we observe the trace estimate

$$
\left\|\left.v\right|_{|x|=2^{2 k_{0}+1}} ; H^{s+1 / 2}(\ldots)\right\| \leq C\left\|v ; H^{s+1}\left(G_{k_{0}+1}\right)\right\| .
$$

4. A priori estimates in step weighted spaces. To find optimal estimates for the perturbed problem $\left(\mathrm{S}_{\varepsilon}\right)$, the Kondratiev spaces as in Section 3.2 are not adequate. This is due to the fact that the differential operator $S_{\varepsilon}$ is not admissible in any neighborhood of infinity in the sense of this theory (see, e.g., [9, p. 99 and p. $241 \mathrm{ff}]$ ). We have to take into account the structure of the differential operator as well as the dependence on the small parameter $\varepsilon$. Following [8], we do this by introducing step weighted spaces. For 
$l \in \mathbb{N}_{0}, \varkappa \leq l$ and $\varepsilon \in(0,1]$ we define $V_{\varkappa, \beta}^{l}(\Omega ; \varepsilon)$ by completing $C_{0}^{\infty}(\bar{\Omega})$ in the norm $\left\|\cdot ; V_{\varkappa, \beta}^{l}(\Omega ; \varepsilon)\right\|$, where

$$
\left\|\varphi ; V_{\varkappa, \beta}^{l}(\Omega ; \varepsilon)\right\|^{2}=\left\|\varphi ; V_{\beta}^{\varkappa}(\Omega)\right\|^{2}+\sum_{h=0}^{l} \int_{\Omega} r^{2(\beta-\varkappa+h)}\left(\frac{\varepsilon}{r}\right)^{2(h-\varkappa)_{+}}\left|\nabla^{h} \varphi\right| d x .
$$

Thus, for $\varkappa=l$, we have $V_{\varkappa, \beta}^{l}(\Omega)=V_{\beta}^{l}(\Omega)$. For $\varkappa<l$, we can rewrite (4.1) as

$$
\left\|\varphi ; V_{\varkappa, \beta}^{l}(\Omega ; \varepsilon)\right\|^{2}=\left\|\varphi ; V_{\beta}^{\varkappa}(\Omega)\right\|^{2}+\sum_{\varkappa<h \leq l} \varepsilon^{2(h-\varkappa)}\left\|r^{\beta} \nabla^{h} \varphi ; L^{2}(\Omega)\right\|^{2} .
$$

Here the weight exponent of $r^{\cdots}$ is the same for all derivatives $\nabla^{h} v$ with $h \geq \varkappa$, in contrast to the Kondratiev norms $\left\|\cdot ; V_{\beta+l-\varkappa}^{l}(\Omega)\right\|$, where the weight exponent increases by 1 with every derivation. If $\varkappa=0$, all derivatives $\nabla^{h} \varphi \in V_{\beta}^{0}(\Omega)$, but accompanied with the factor $\varepsilon^{h}$. For $\varkappa<0$, we have

$$
\left\|\varphi ; V_{\varkappa, \beta}^{l}(\Omega ; \varepsilon)\right\|^{2}=\left\|\varphi ; V_{\beta}^{\varkappa}(\Omega)\right\|^{2}+\varepsilon^{-2 \varkappa}\left\|\varphi ; V_{0, \beta}^{l}(\Omega ; \varepsilon)\right\|^{2} .
$$

In any case $V_{\varkappa, \beta}^{l}(\Omega ; \varepsilon) \subset H_{l o c}^{l}(\bar{\Omega})$. Since $\partial \Omega$ is compact, there are the continuous trace operators $\left.\varphi \rightarrow \partial_{n}^{h} \varphi\right|_{\partial \Omega}$, where $h=0,1$ and $l \geq 1+h$ as in Section 2 with $\varepsilon$-independent bounds for their norms. This means, if we supply $H^{l-h-1 / 2}(\partial \Omega)$ with the norm of $\mathcal{H}_{\varkappa-h-1 / 2}^{l-h-1 / 2}(\partial \Omega ; \varepsilon)$ as in Section 2 , then

$$
\left\|\partial_{n}^{h} \varphi ; \mathcal{H}_{\varkappa-h-1 / 2}^{l-h-1 / 2}(\partial \Omega ; \varepsilon)\right\| \leq C\left\|\varphi ; V_{\varkappa, \beta}^{l}(\Omega ; \varepsilon)\right\|
$$

with $C$ independent on $\varepsilon \in(0,1]$ and $\beta \in \mathbb{R}$. The following estimates on homogeneous partial differential operators are validated by elementary calculations.

Proposition 4.1. Assume $l \in \mathbb{N}, \varkappa \leq l, \beta \in \mathbb{R}, \mu \in[0,1]$ and $\alpha \in \mathbb{N}_{0}^{3}$ with $|\alpha| \leq l$. Then for any $\varphi \in V_{\varkappa, \beta}^{l}(\Omega ; \varepsilon)$,

$$
\left\|\partial^{\alpha} \varphi ; V_{\varkappa-|\alpha|, \beta}^{l-\alpha}(\Omega ; \varepsilon)\right\| \leq C\left\|\varphi ; V_{\varkappa, \beta}^{l}(\Omega ; \varepsilon)\right\|
$$

and, if $\varkappa+\mu \leq l-|\alpha|$,

$$
\left\|\varepsilon^{|\alpha|+\mu} \partial^{\alpha} \varphi ; V_{\varkappa+\mu, \beta}^{l-|\alpha|}(\Omega ; \varepsilon)\right\| \leq C\left\|\varphi ; V_{\varkappa, \beta}^{l}(\Omega ; \varepsilon)\right\| .
$$

To problem $\left(\mathrm{S}_{\varepsilon}\right)$ we assign the following natural domain and range (compare (3.11) and (3.12)) for $l \geq \varkappa+1$ :

$$
\begin{aligned}
\mathscr{D}_{\varkappa, \beta}^{l} V(\Omega ; \varepsilon)= & V_{\varkappa+1, \beta}^{l+1}(\Omega ; \varepsilon)^{3} \times V_{\varkappa, \beta}^{l+1}(\Omega ; \varepsilon), \\
\mathscr{R}_{\varkappa, \beta}^{l} V(\Omega ; \varepsilon)= & V_{\varkappa-1, \beta}^{l-1}(\Omega ; \varepsilon)^{3} \times V_{\varkappa, \beta}^{l-1}(\Omega ; \varepsilon) \times \\
& \times H_{\varkappa+1 / 2}^{l+1 / 2}(\partial \Omega ; \varepsilon)^{3} \times H_{\varkappa-3 / 2}^{l-1 / 2}(\partial \Omega ; \varepsilon) .
\end{aligned}
$$

From $(4.3)-(4.5)$, we obtain that the operator related to problem $\left(\mathrm{S}_{\varepsilon}\right)$

$$
\mathcal{A}_{\varkappa, \beta}^{l, \varepsilon}: \mathscr{D}_{\varkappa, \beta}^{l} V(\Omega ; \varepsilon) \ni u \rightarrow\left(S_{\varepsilon} u, B_{\varepsilon} u\right) \in \mathscr{R}_{\varkappa, \beta}^{l}(\Omega ; \varepsilon)
$$

has a norm bounded independent of $\varepsilon \in(0,1]$.

We proceed with an $\varepsilon$-independent a priori estimate for solutions to problem $\left(\mathrm{S}_{\varepsilon}\right)$.

Lemma 4.2. Let $\varkappa \in[0,3 / 2), h \in \mathbb{N}, l \geq \varkappa+1$ and $\beta \in \mathbb{R}$ be given. Assume further $u^{\varepsilon}=\left(v^{\varepsilon}, p^{\varepsilon}\right)$ with $v^{\varepsilon} \in H_{l o c}^{l+1}(\bar{\Omega})^{3} \cap V_{\beta-\varkappa-1}^{0}(\Omega)^{3}, p^{\varepsilon} \in H_{l o c}^{l+1}(\Omega) \cap V_{\beta-\varkappa}^{0}(\Omega)$ is a solution 
to $\left(\mathrm{S}_{\varepsilon}\right)$ with $(f, g) \in \mathscr{R}_{\varkappa, \beta}^{l} V(\Omega ; \varepsilon)$. Then $u^{\varepsilon} \in \mathscr{D}_{\varkappa, \beta}^{l} V(\Omega ; \varepsilon)$ and $\left\|u^{\varepsilon} ; \mathscr{D}_{\varkappa, \beta}^{l} V(\Omega ; \varepsilon)\right\| \leq C\left(\left\|(f, g) ; \mathscr{R}_{\varkappa, \beta}^{l} V(\Omega ; \varepsilon)\right\|+\left\|v^{\varepsilon} ; V_{\beta-\varkappa-1}^{0}(\Omega)^{3}\right\|+\left\|p^{\varepsilon} ; V_{\beta-\varkappa}^{0}(\Omega)\right\|\right)$.

The constant $C$ is independent of $u^{\varepsilon}$, the data $(f, g)$ and the parameter $\varepsilon \in(0,1]$.

Proof. Let $k_{0}$ and $G_{k}, G_{k}^{\prime}, \Xi, \Xi^{\prime}$ be defined as in the proof of Theorem 3.2. On $G_{k_{0}}$ we apply (2.2) and obtain

$$
\left\|u^{\varepsilon} ; \mathscr{D}_{\varkappa}^{l} H\left(G_{k_{0}}^{\prime} ; \varepsilon\right)\right\|^{2} \leq C\left(\left\|(f, g) ; \mathscr{D}_{\varkappa}^{l} H\left(G_{k_{0}} ; \varepsilon\right)\right\|^{2}+\left\|u^{\varepsilon} ; L^{2}\left(G_{k_{0}}\right)\right\|^{2}\right) .
$$

For $k>k_{0}$, we obtain from $(3.7)_{1}$ :

$$
\begin{aligned}
-2^{-2 k} \Delta_{\xi} \hat{v}_{k}^{\varepsilon}+2^{-k} \nabla_{\xi} \hat{p}_{k}^{\varepsilon} & =\hat{f}_{k}^{\prime}, \\
-\varepsilon^{2} 2^{-2 k} \Delta_{\xi} \hat{p}_{k}^{\varepsilon}+2^{-k} \operatorname{div} \hat{v}_{k}^{\varepsilon} & =\hat{f}_{4, k}
\end{aligned}
$$

on $\Xi$. Thus, $\left(\hat{v}_{k}^{\varepsilon}, 2^{k} \hat{p}_{k}^{\varepsilon}\right)$ solves the problem

$$
S_{\tilde{\varepsilon}, \xi}\left(\hat{v}_{k}^{\varepsilon}, 2^{k} \hat{p}_{k}^{\varepsilon}\right)=\left(2^{2 k} \hat{f}_{k}^{\prime}, 2^{k} \hat{f}_{4, k}\right)
$$

on $\Xi$ with the new small parameter $\varepsilon_{k}=2^{-k} \varepsilon$. The interior estimate (2.2) leads to

$$
\begin{aligned}
\left\|\hat{v}_{k}^{\varepsilon} ; H_{\varkappa+1}^{l+1}\left(\Xi^{\prime} ; \varepsilon_{k}\right)\right\|^{2}+ & \left\|2^{k} \hat{p}_{k}^{\varepsilon} ; H_{\varkappa}^{l+1}\left(\Xi^{\prime} ; \varepsilon_{k}\right)\right\|^{2} \\
& \leq C\left(\left\|2^{2 k} \hat{f}_{k}^{\prime} ; H_{\varkappa-1}^{l-1}\left(\Xi ; \varepsilon_{k}\right)\right\|^{2}+\left\|2^{2 k} \hat{f}_{4, k} ; H_{\varkappa}^{l-1}\left(\Xi ; \varepsilon_{k}\right)\right\|^{2}\right. \\
& \left.+\left\|\hat{v}_{k}^{\varepsilon} ; L^{2}(\Xi)\right\|^{2}+\left\|2^{k} \hat{p}_{k}^{\varepsilon} ; L^{2}(\Xi)\right\|^{2}\right),
\end{aligned}
$$

where $C$ is a constant independent of $k$ and $\varepsilon$. Observe that due to the transformation of the small parameter $\varepsilon$, for example

$$
\left\|\hat{v}_{k}^{\varepsilon} ; H_{\varkappa+1}^{l+1}\left(\Xi^{\prime} ; \varepsilon_{k}\right)\right\|^{2}=\sum_{k=0}^{l+1}\left(\varepsilon 2^{-k}\right)^{2(h-\varkappa-1)+}\left\|\nabla_{\xi}^{h} \hat{v}^{\varepsilon} ; L^{2}\left(\Xi^{\prime}\right)\right\|^{2},
$$

the other expressions in (4.8) are calculated correspondingly. We multiply (4.8) by $2^{2 k(\beta-\varkappa-1)}$, sum over (4.7) and (4.8) $k$ with $k>k_{0}$, then Lemma 3.1 gives the desired a priori estimate.

The next step is an investigation of solutions to the Stokes problem $\left(\mathrm{S}_{0}\right)$ in $V_{\varkappa, \beta}^{l}(\Omega ; \varepsilon)$ spaces. The difference from the results of Theorem 3.2 is the following, very roughly speaking: for data in Kondratiev spaces the asymptotic decay with $r \rightarrow \infty$ of the data as well as for the solutions increases with each derivative. In $V_{\varkappa, \beta}^{l}(\Omega ; \varepsilon)$-spaces the asymptotic behavior of the data's derivatives remains the same for derivatives of sufficiently high order, the same we can anticipate for the solutions. On the other hand, if e.g. $f^{\prime} \in H^{l}(\Omega)$, and $f_{4}=0$ in problem $\left(\mathrm{S}_{0}\right)$, we cannot expect a solution with $v \in H^{l+2}(\Omega), p \in H^{l+1}(\Omega)$, but only $\nabla^{2} v \in H^{l}(\Omega), \nabla p \in H^{l}(\Omega)$, while $v \in V_{0}^{2}(\Omega)$, and $p \in V_{0}^{1}(\Omega)$ (see, e.g., [13]). For $l \in \mathbb{N}$ and $s \in[0, l]$ we put

$$
\begin{aligned}
& \mathbf{D}_{\varkappa, \beta}^{l} V(\Omega ; \varepsilon)=V_{\varkappa+1, \beta}^{l+1}(\Omega ; \varepsilon)^{3} \times V_{\varkappa, \beta}^{l}(\Omega ; \varepsilon) \\
& \mathbf{R}_{\varkappa, \beta}^{l} V(\Omega ; \varepsilon)=V_{\varkappa-1, \beta}^{l-1}(\Omega ; \varepsilon)^{3} \times V_{\varkappa, \beta}^{l}(\Omega ; \varepsilon) \times \mathcal{H}_{\varkappa+1 / 2}^{l+1 / 2}(\partial \Omega ; \varepsilon)^{3} .
\end{aligned}
$$

Proposition 4.3. Let $|\beta-\varkappa|<1 / 2$. Then the mapping $u \rightarrow\left(S_{0} u, B_{0} u\right)$ defines the isomorphism $\mathbf{A}_{\varkappa, \beta}^{l}: \mathbf{D}_{\varkappa, \beta}^{l} V(\Omega ; \varepsilon) \rightarrow \mathbf{R}_{\varkappa, \beta}^{l} V(\Omega ; \varepsilon)$, where the norm of $\mathbf{A}_{\varkappa, \beta}^{l}$ as well as the norm of $\left(\mathbf{A}_{\varkappa, \beta}^{l}\right)^{-1}$ are majorized by a constant independent of $\varepsilon \in(0,1]$. 
Proof. The continuity of $\mathbf{A}_{\varkappa, \beta}^{l}$ with $\varepsilon$-independent bounds for the norms follows directly from the definition of the spaces. Since (compare (3.11), (3.12))

$$
\left\|\left(f, g^{\prime}\right) ; \mathscr{R}_{\beta}^{\varkappa} V(\Omega)\right\| \leq\left\|\left(f, g^{\prime}\right) ; \mathbf{R}_{\varkappa, \beta}^{l} V(\Omega ; \varepsilon)\right\|,
$$

Theorem 3.2 ensures the existence of a unique solution $u \in \mathscr{D}_{\beta}^{\varkappa} V(\Omega)$ to problem $\left(\mathrm{S}_{0}\right)$, together with the estimate

$$
\left\|u ; \mathscr{D}_{\beta}^{\varkappa} V(\Omega)\right\| \leq C\left\|\left(f, g^{\prime}\right) ; \mathscr{R}_{\beta}^{\varkappa} V(\Omega)\right\| .
$$

Moreover, $v \in H_{l o c}^{l+1}(\bar{\Omega}), p \in H_{l o c}^{l}(\bar{\Omega})$. It remains to estimate the terms

$$
\begin{array}{cl}
\varepsilon^{q-\varkappa-1}\left\|r^{\beta} \nabla^{q} v ; L^{2}(\Omega)\right\|, & {[\varkappa+1]<q \leq l+1,} \\
\varepsilon^{q-\varkappa}\left\|r^{\beta} \nabla^{q} p ; L^{2}(\Omega)\right\|, & {[\varkappa]<q \leq l .}
\end{array}
$$

Since $0 \notin \bar{\Omega}$, the space $V_{\gamma}^{0}(\Omega)$ is continuously embedded into $V_{\gamma-\delta}^{0}(\Omega)$ for any $\gamma \in \mathbb{R}$, and $\delta \geq 0$, therefore, we have for $q=[\varkappa]+1, \ldots, l$

$$
\left\|u ; V_{\beta-q-1}^{0}(\Omega)^{3} \times V_{\beta-q}^{0}(\Omega)\right\| \leq C\left\|u ; V_{\beta-\varkappa-1}^{0}(\Omega)^{3} \times V_{\beta-s}^{0}(\Omega)\right\| .
$$

From the definition (4.2) of the $V_{\varkappa, \beta^{l}}^{l}$ norms and the trace norms in Section 2, we also get

$$
\left\|\left(f, g^{\prime}\right) ; \mathscr{R}_{\beta}^{q} V(\Omega)\right\| \leq C \varepsilon^{-(q-\varkappa)}\left\|\left(f, g^{\prime}\right) ; \mathbf{R}_{\varkappa, \beta}^{l} V(\Omega ; \varepsilon)\right\|,
$$

where $C$ is independent of $\varepsilon \in(0,1]$. Note that on the left-hand side we have just the Kondratiev space, where the highest derivatives $\nabla^{q-1} f^{\prime}$ and $\nabla^{q} f_{4} \in V_{\beta}^{0}(\Omega)$.

We multiply the a priori estimate (3.15) for the Stokes system by $\varepsilon^{q-\varkappa}$, then use $\varepsilon \leq 1$ and $q-\varkappa>0,(4.14),(4.12)$ and (4.15) and arrive at

$$
\begin{aligned}
& \varepsilon^{q-\varkappa}\left\|u ; V_{\beta}^{q+1}(\Omega)^{3} \times V_{\beta}^{q}(\Omega)\right\| \\
& \leq C \varepsilon^{q-\varkappa}\left(\left\|\left(f, g^{\prime}\right) ; \mathscr{R}_{\beta}^{q} V(\Omega)\right\|+\left\|u ; V_{\beta-q-1}^{0}(\Omega)^{3} \times V_{\beta-q}^{0}(\Omega)\right\|\right) \\
& \leq C\left(\left\|\left(f, g^{\prime}\right) ; \mathbf{R}_{\varkappa, \beta}^{l} V(\Omega ; \varepsilon)\right\|+\varepsilon^{q-\varkappa}\left\|u ; V_{\beta-q-1}^{0}(\Omega)^{3} \times V_{\beta-q}^{0}(\Omega)\right\|\right) \\
& \leq C\left(\left\|\left(f, g^{\prime}\right) ; \mathbf{R}_{\varkappa, \beta}^{l} V(\Omega ; \varepsilon)\right\|+\varepsilon^{q-\varkappa}\left\|u ; V_{\beta-\varkappa-1}^{0}(\Omega)^{3} \times V_{\beta-\varkappa}^{0}(\Omega)\right\|\right) \\
& \leq C\left(\left\|\left(f, g^{\prime}\right) ; \mathbf{R}_{\varkappa, \beta}^{l} V(\Omega ; \varepsilon)\right\|+\varepsilon^{q-\varkappa}\left\|\left(f, g^{\prime}\right) ; \mathscr{R}_{\beta}^{\varkappa} V(\Omega)\right\|\right) \\
& \leq C\left\|\left(f, g^{\prime}\right) ; \mathbf{R}_{\varkappa, \beta}^{l}(\Omega ; \varepsilon)\right\|,
\end{aligned}
$$

for the last inequality we used $\varepsilon \leq 1$ again together with the inequality (4.11). In the whole chain the constants $C$ are independent of $\varepsilon$. The left-hand side majorizes the terms in (4.13), thus we have $\left\|\left(\mathbf{A}_{\varkappa, \beta}^{l}\right)^{-1}\right\| \leq C$ independent of $\varepsilon$.

5. Asymptotics of the solutions to problem $\left(\mathbf{S}_{\varepsilon}\right)$. Let $u^{\varepsilon} \in \mathscr{D}_{\varkappa, \beta}^{l} V(\Omega ; \varepsilon)$ be a solution to $\left(\mathrm{S}_{\varepsilon}\right)$ with $(f, g) \in \mathscr{R}_{\varkappa, \gamma}^{l} V(\Omega ; \varepsilon)$ where

$$
l \in \mathbb{N}, \gamma=\beta+\delta, \delta \geq 0, \varkappa \neq 1 / 2, \varkappa \leq l-1, \gamma-\beta+1 / 2 \notin \mathbb{Z}, \varkappa-\beta+1 / 2 \notin \mathbb{Z} .
$$

This means that the data of the problem have a stronger asymptotic decay as $r \rightarrow \infty$ than the solution lets expect. The aim now is to prove a result analogous to part (iv) of Theorem 3.2. Since for a solution to the homogeneous Stokes problem, the pressure is a harmonic function, any power solution $U$ of (3.14) solves the problem $S_{\varepsilon} U=0$ in $\mathbb{R}^{3} \backslash\{0\}$. Moreover, if $U$ is of the form (3.13), then $\left.U\right|_{\Omega} \in \mathscr{D}_{\varkappa, \beta}^{l} V(\Omega ; \varepsilon)$ as long as $\int_{1}^{\infty} r^{2 \lambda} r^{2(\beta-\varkappa-1)} r^{2} d r<\infty$, i.e. $\beta-\varkappa<-\lambda-1 / 2$. It is not surprising that the power 
solutions of $\left(\mathrm{S}_{0}\right)$ appear in the asymptotics of $u^{\varepsilon}$. The amazing point is that they describe already the complete asymptotic expansion.

The proof of this result requires a subtle play with embedding inequalities between Kondratiev and step weighted spaces, and the known results for the asymptotics of the solutions to $\left(\mathrm{S}_{0}\right)$. To this end, we observe that $u^{\varepsilon}$ solves the Stokes problem

$$
-\Delta v^{\varepsilon}+\nabla p^{\varepsilon}=f^{\prime}, \quad \operatorname{div} v^{\varepsilon}=f_{4}+\varepsilon^{2} \Delta p^{\varepsilon},\left.\quad v^{\varepsilon}\right|_{\partial \Omega}=g^{\prime} .
$$

The main point here is to find the common Sobolev space for the right-hand side of the divergence equation. At a first glance, we find $f_{4} \in V_{\gamma}^{\varkappa}(\Omega) \subset V_{\gamma-\varkappa}^{0}(\Omega)$, while $\varepsilon^{2} \Delta v^{\varepsilon} \in$ $V_{\beta}^{0}(\Omega)$. Since $0 \notin \bar{\Omega}$, we have $V_{\beta}^{0}(\Omega) \subset V_{\beta-(\varkappa-\delta)}^{0}(\Omega)$, if $\varkappa-\delta \geq 0$. For the moment this leads to a restriction for the gap between the weight exponents $\beta$ and $\gamma$, which we have to overcome. We also emphasize an elementary, but fundamental property of the $V_{\beta}^{\varkappa}(\Omega)$ spaces: the decay at infinity is determined by the difference $\beta-\varkappa$. Thus $\varphi \in V_{\beta}^{\varkappa-\delta}(\Omega)$ means that $\varphi$ has less smoothness than $V_{\beta}^{\varkappa}(\Omega)$-functions, but a stronger decay at infinity. On the other hand, the asymptotic expansion of a solution to $\left(\mathrm{S}_{0}\right)$ depends only on the decay at infinity of the data and the solution, but not on their smoothness properties.

We collect the precise conclusions about the right-hand sides to problem (5.2) in the next lemma. Before this we recall some inequalities in Kondratiev spaces and step weighted spaces, which can be easily derived from their definitions. Due to (4.2), we have for $\varkappa \leq l, l \in \mathbb{N}_{0}, \beta \in \mathbb{R}$ and $\varphi \in V_{\varkappa, \beta}^{l} V(\Omega ; \varepsilon)$ :

$$
\left\|\varphi ; V_{\beta}^{\varkappa}(\Omega)\right\| \leq\left\|\varphi ; V_{\varkappa, \beta}^{l} V(\Omega ; \varepsilon)\right\| .
$$

Furthermore, if $\delta \geq 0$ in addition, then

$$
\left\|\varphi ; V_{\varkappa-\delta, \beta}^{l}(\Omega ; \varepsilon)\right\| \leq C\left\|\varphi ; V_{\varkappa, \beta+\delta}^{l}(\Omega ; \varepsilon)\right\| .
$$

As for the last inequality, we can compare the stable parts of the norms and the $\varepsilon$ dependent parts directly. From the definition of the Kondratiev norms we see

$$
\left\|\varphi ; V_{\beta}^{\varkappa-\delta}(\Omega)\right\| \leq C\left\|\varphi ; V_{\beta+\delta}^{\varkappa}(\Omega)\right\| .
$$

If $\varkappa-\delta \in \mathbb{N}_{0}$, this is evident, otherwise we can use Lemma 3.1 and interpolation inequalities. Since $\varepsilon \in(0,1]$, and $r \geq r_{0}=\max \{|x|: x \in \partial \Omega\}>0$, we obtain for $h>\varkappa-\delta$ :

$$
\varepsilon^{h-(\varkappa-\delta)}\left\|r^{\beta} \nabla^{h} \varphi ; L^{2}(\Omega)\right\| \leq C \varepsilon^{h-\varkappa}\left\|r^{\beta+\delta} \nabla^{h} \varphi ; L^{2}(\Omega)\right\|,
$$

which finally shows (5.4).

If, in addition, $\varkappa \neq 1 / 2, \varkappa-\delta \neq 1 / 2$, then from the definition of the trace norms in Section 2, we also obtain

$$
\left\|\varphi_{\mid \partial \Omega} ; \mathcal{H}_{\varkappa-\delta-1 / 2}^{l-1 / 2}(\partial \Omega ; \varepsilon)\right\| \leq C\left\|\varphi ; \mathcal{H}_{\varkappa-1 / 2}^{l-1 / 2}(\partial \Omega ; \varepsilon)\right\| .
$$

LEMmA 5.1. Let $l, \gamma, \beta$ and $\delta$ fulfil (5.1) and assume in addition, that $\varkappa-\delta \geq 0$, but $\neq 1 / 2$, then for $(f, g) \in \mathscr{R}_{\varkappa, \gamma}^{l} V(\Omega ; \varepsilon)$ it follows that

$$
\left\|\left(f, g^{\prime}\right) ; \mathscr{R}_{\beta}^{\varkappa-\delta} V(\Omega)\right\| \leq C\left\|\left(f, g^{\prime}\right) ; \mathscr{R}_{\varkappa-\delta, \beta}^{l} V(\Omega ; \varepsilon)\right\| \leq C\left\|(f, g) ; \mathscr{R}_{\varkappa, \gamma}^{l} V(\Omega ; \varepsilon)\right\| .
$$

For $p \in V_{\varkappa, \beta}^{l+1}(\Omega)$,

$$
\begin{aligned}
&\left\|\varepsilon^{2} \Delta p ; V_{0, \beta}^{l-1}(\Omega ; \varepsilon)\right\| \leq C \varepsilon^{\varkappa}\left\|p ; V_{\varkappa, \beta}^{l+1}(\Omega ; \varepsilon)\right\|, \\
&\left\|\varepsilon^{2} \Delta p ; V_{\beta}^{\varkappa-\delta}(\Omega)\right\| \leq C\left\|p ; V_{\varkappa, \beta}^{l+1}(\Omega ; \varepsilon)\right\| .
\end{aligned}
$$


Proof. Estimate (5.7) immediately follows from (5.4) and (5.6). As for the other two inequalities, we observe that from definition (4.1) we have

$$
\mathfrak{P}:=\sum_{q=0}^{l+1} \int_{\Omega} r^{2(\beta-\varkappa+q)}\left(\frac{\varepsilon}{r}\right)^{2(q-\varkappa)_{+}}\left|\nabla^{q} p(x)\right|^{2} d x \leq\left\|p ; V_{\varkappa, \beta}^{l+1}(\Omega ; \varepsilon)\right\|^{2} .
$$

Since $\varkappa<3 / 2$, and, therefore, $2+q-\varkappa>0$ for $q=0,1, \ldots, l-1$ we obtain

$$
\begin{aligned}
& \varepsilon^{-2 \varkappa}\left\|\varepsilon^{2} \Delta p ; V_{0, \beta}^{l-1}(\Omega ; \varepsilon)\right\|^{2} \\
& =\sum_{q=0}^{l-1} \int_{\Omega} r^{2(\beta-\varkappa+2+q)}\left(\frac{\varepsilon}{r}\right)^{2(2+q-\varkappa)}\left\|\nabla^{q} \Delta p(x)\right\|^{2} d x \leq C \mathfrak{P} .
\end{aligned}
$$

This already proves (5.8).

Now let $G_{k}, k \geq k_{0}$ be defined as in Lemma 3.1. The square of the left-hand side of (5.9) is equivalent to

$$
\left\|\varepsilon^{2} \Delta p ; H^{\varkappa-\delta}\left(G_{k_{0}}\right)\right\|^{2}+\sum_{k>k_{0}}\left(2^{k}\right)^{2(\beta-\varkappa+\delta)+3}\left\|2^{-2 k} \varepsilon^{2} \Delta_{\xi} \hat{p}_{k} ; H^{\varkappa-\delta}(\Xi)\right\|^{2},
$$

while the left-hand side of (5.10) is equivalent to

$$
\begin{aligned}
\varepsilon^{-2 \varkappa}\left\|\varepsilon^{2} \Delta p ; H_{0}^{l-1}\left(G_{k_{0}}\right)\right\|^{2}+ & \\
& \sum_{k>k_{0}} \sum_{q=0}^{l-1} \varepsilon^{2(q-\varkappa)}\left(2^{k}\right)^{2(\beta-q)+3}\left\|\nabla_{\xi}^{q}\left(\varepsilon^{2} 2^{-2 k} \Delta_{\xi} \hat{p}_{k}\right) ; L^{2}(\Xi)\right\|^{2} .
\end{aligned}
$$

Clearly, we have

$$
\left\|\varepsilon^{2} \Delta p ; H^{\varkappa-\delta}\left(G_{k_{0}}\right)\right\| \leq \varepsilon^{-\varkappa}\left\|\varepsilon^{2} \Delta p ; H^{l-1}\left(G_{k_{0}}\right)\right\|,
$$

since $\varkappa-\delta \leq l-1$ and $\varkappa \geq 0$. It remains to compare the summands with $k>k_{0}$ in (5.11) and (5.12). Let us first consider $\varkappa-\delta=0,1$. If $\varkappa-\delta=0$, then the $k$-th summand in (5.11) is majorized by the $k$-th summand of (5.12), since $\varepsilon^{-\varkappa} \geq 1$, the same is true, if $\varkappa-\delta=1$, since then $\varepsilon^{-\varkappa+1} \geq 1$. If $\varkappa-\delta$ is non-integer, then we use an interpolation argument: We put $\psi=\left(2^{k}\right)^{\beta-2+3 / 2} \varepsilon^{2} \Delta_{\xi} \hat{p}_{k}$ and recall the interpolation inequality

$$
\left\|\psi ; H^{\varkappa-\delta}(\Xi)\right\|^{2} \leq C\left\|\psi ; H^{m}(\Xi)\right\|^{2(\varkappa-\delta) / m}\left\|\psi ; L^{2}(\Xi)\right\|^{2-2(\varkappa-\delta) / m}
$$

with $m=1$ for $\varkappa-\delta<1$ and $m=2$ for $\varkappa-\delta \in[1,3 / 2)$. Multiplying this inequality by $\left(2^{2 k}\right)^{-(\varkappa-\delta)}$ and applying Young's inequality, we find that

$$
\begin{aligned}
\left(2^{2 k}\right)^{-(\varkappa-\delta)}\left\|\psi ; H^{\varkappa-\delta}(\Xi)\right\| \\
\leq C\left(\left(2^{2 k}\right)^{-(\varkappa-\delta)}\left\|\psi ; H^{m}(\Xi)\right\|^{2(\varkappa-\delta) / m} \varepsilon^{2(\varkappa-\delta)(1-(\varkappa-\delta) / m)}\right) \times \\
\quad\left(\left\|\psi ; L^{2}(\Xi)\right\|^{2-2(\varkappa-\delta) / m} \varepsilon^{-2(\varkappa-\delta)(1-(\varkappa-\delta) / m)}\right) \\
\leq C\left(\left(2^{2 k}\right)^{-m} \varepsilon^{2(m-(\varkappa-\delta))}\left\|\psi ; H^{m}(\Xi)\right\|^{2}+\varepsilon^{-2(\varkappa-\delta)}\left\|\psi ; L^{2}(\Xi)\right\|^{2}\right),
\end{aligned}
$$

where $C$ depends on $\varkappa-\delta$, but not on $\varepsilon \in(0,1]$. Thus, $\varepsilon^{-(\varkappa-\delta)} \leq \varepsilon^{-\varkappa}$ we obtain that the $k$-th summand of (5.11) can be estimated by the $k$-th summand of (5.12) multiplied with a constant $C$. This yields (5.9).

With Lemma 5.1 at hand, we can prove the result on the asymptotics of the solutions $u^{\varepsilon}$. 
TheOREM 5.2. Let $\varkappa \in[0,3 / 2), l, \beta, \gamma$ fulfill (5.1). Suppose $u^{\varepsilon} \in \mathscr{D}_{\varkappa, \beta}^{l} V(\Omega ; \varepsilon)$ is a solution to $\left(S_{\varepsilon} u^{\varepsilon}, B_{\varepsilon} u^{\varepsilon}\right)=(f, g)$, where $(f, g) \in \mathscr{R}_{\varkappa, \gamma}^{l} V(\Omega ; \varepsilon)$. Then $u^{\varepsilon}$ admits the asymptotic representation

$$
u^{\varepsilon}=\sum_{j=1}^{J} b_{j} U_{j}+\tilde{u}^{\varepsilon} .
$$

Here $U_{j}=\left(r^{\lambda} \mathcal{U}_{j}(\phi), r^{\lambda-1} \mathcal{P}_{j}(\phi)\right)$ is a power solution to the Stokes system as in $(3.17)$, and $\lambda$ again is an integer in the interval $I_{0}=(\varkappa-\gamma-1 / 2, \varkappa-\beta-1 / 2)$. The corresponding estimate holds with a constant $C$ independent of $\varepsilon$ :

$$
\left\|\tilde{u}^{\varepsilon} ; \mathscr{D}_{\varkappa, \gamma}^{l} V(\Omega ; \varepsilon)\right\|+\sum_{j=1}^{J}\left|b_{j}\right| \leq C\left(\left\|(f, g) ; \mathscr{R}_{\varkappa, \gamma}^{l} V(\Omega ; \varepsilon)\right\|+\left\|u^{\varepsilon} ; \mathscr{D}_{\varkappa, \beta}^{l} V(\Omega ; \varepsilon)\right\|\right) .
$$

If $I_{0}$ contains no integer, then the sums in (5.13) and (5.14) are void.

Proof. Step 1. Let us first assume that $\gamma-\beta=\delta \in(0, \varkappa) \backslash\{1 / 2\}$. From Lemma 5.1 we obtain $\left(f^{\prime}, f_{4}+\varepsilon^{2} \Delta p^{\varepsilon}, g^{\prime}\right) \in \mathscr{R}_{\beta}^{\varkappa-\delta} V(\Omega)$, while for the solution $u^{\varepsilon}$ we use (5.3) to see

$$
\left\|u^{\varepsilon} ; \mathscr{D}_{\beta-\delta}^{\varkappa-\delta} V(\Omega)\right\| \leq C\left\|u^{\varepsilon} ; \mathscr{D}_{\beta}^{\varkappa} V(\Omega)\right\| \leq C\left\|u^{\varepsilon} ; \mathscr{D}_{\varkappa, \beta}^{l} V(\Omega ; \varepsilon)\right\| .
$$

Observe that $I_{0}=(\varkappa-\delta-\beta-1 / 2, \varkappa-\delta-(\beta-\delta)-1 / 2)$. Theorem 3.2 (iv) provides us with the representation (5.13) and the estimate

$$
\begin{aligned}
\sum_{j=1}^{J}\left|b_{j}\right|+\| \tilde{u}^{\varepsilon} ; & \mathscr{D}_{\beta}^{\varkappa-\delta} V(\Omega) \| \leq C\left(\left\|\left(f^{\prime}, f_{4}+\varepsilon^{2} \Delta p^{\varepsilon}, g^{\prime}\right) ; \mathscr{R}_{\beta}^{\varkappa-\delta} V(\Omega)\right\|\right. \\
& \left.\left.+\| u^{\varepsilon} ; V_{\beta-\delta-(\varkappa-\delta)}^{0} \Omega\right)^{3} \times V_{\beta-\delta-(\varkappa-\delta)}^{0}(\Omega) \|\right) \\
\leq & C\left(\left\|(f, g) ; \mathscr{R}_{\varkappa, \gamma}^{l}(\Omega ; \varepsilon)\right\|+\left\|v^{\varepsilon} ; V_{\beta-\varkappa-1}^{0}(\Omega)\right\|+\left\|p^{\varepsilon} ; V_{\beta-\varkappa}^{0}(\Omega)\right\|\right),
\end{aligned}
$$

here we used (3.18), Lemma 5.1 and (5.15). Since $S_{\varepsilon} U_{j}=0$, we obtain

$$
S_{\varepsilon} \tilde{u}^{\varepsilon}=f \text { in } \Omega, \quad B_{\varepsilon} \tilde{u}^{\varepsilon}=\tilde{g}:=g-B_{\varepsilon} \sum_{j=1}^{J} b_{j} U_{j} \text { on } \partial \Omega .
$$

Moreover, $U_{j}$ are fixed functions and the boundary $\partial \Omega$ is compact, thus

$\left\|(f, \tilde{g}) ; \mathscr{R}_{\varkappa, \gamma}^{l} V(\Omega ; \varepsilon)\right\| \leq C\left(\left\|(f, g) ; \mathscr{R}_{\varkappa, \gamma}^{l} V(\Omega ; \varepsilon)\right\|+\sum_{j=1}^{J}\left|b_{j}\right|\right) \leq C\left\|(f, g) ; \mathscr{R}_{\varkappa, \gamma}^{l}(\Omega ; \varepsilon)\right\|$.

From the definition of the norms it follows again that

$$
\begin{aligned}
& \left\|\tilde{v}^{\varepsilon} ; V_{\gamma-\varkappa-1}^{0}(\Omega)\right\|+\left\|\tilde{p}^{\varepsilon} ; V_{\gamma-\varkappa}^{0}(\Omega)\right\| \\
& \quad=\left\|\tilde{v}^{\varepsilon} ; V_{\beta-(\varkappa-\delta)-1}^{0}(\Omega)\right\|+\left\|\tilde{p}^{\varepsilon} ; V_{\beta-(\varkappa-\delta)}^{0}(\Omega)\right\| \leq\left\|\tilde{u} ; \mathscr{D}_{\beta}^{\varkappa-\delta} V(\Omega)\right\| .
\end{aligned}
$$

We apply the a priori estimate of Lemma 4.2 and find that

$$
\left\|\tilde{u}^{\varepsilon} ; \mathscr{D}_{\varkappa, \gamma}^{l} V(\Omega ; \varepsilon)\right\| \leq C\left(\left\|(f, \tilde{g}) ; \mathscr{R}_{\varkappa, \gamma}^{l} V(\Omega ; \varepsilon)\right\|+\left\|\tilde{v}^{\varepsilon} ; V_{\gamma-\varkappa-1}^{0}(\Omega)\right\|+\left\|\tilde{p}^{\varepsilon} ; V_{\gamma-\varkappa}^{0}(\Omega)\right\|\right) \text {. }
$$

Combining (5.16) and (5.17) with the last inequality leads to (5.14).

Step 2. Now we permit $\delta=\gamma-\beta>\varkappa$ for the gap. We choose $N$ large enough, such that $\tilde{\delta}=\delta / N \in(0, \varkappa) \backslash\{1 / 2\}$. Due to $0 \notin \bar{\Omega}$, it follows that

$$
\left\|(f, g) ; \mathscr{R}_{\varkappa, \beta+k \tilde{\delta}}^{l} V(\Omega ; \varepsilon)\right\| \leq C\left\|(f, g) ; \mathscr{R}_{\varkappa, \gamma}^{l} V(\Omega ; \varepsilon)\right\|
$$


for $k=1, \ldots, N$. We apply the first step with $\gamma$ replaced by $\beta+\tilde{\delta}$ and find the representation (5.13) (recall that the sum may be void), where $\left\|\tilde{u}^{\varepsilon} ; \mathscr{R}_{\varkappa, \beta+\tilde{\delta}}^{l} V(\Omega ; \varepsilon)\right\|$ may be estimated by the right-hand side of (5.14) with the help of (5.18) for $k=1, \ldots, N$. Iterated utilization of this argument, first time applied to $\tilde{u}^{\varepsilon}, \beta$ replaced by $\beta+k \tilde{\delta}$, and $\gamma$ by $\beta+(k+1) \tilde{\delta}$, for $k=1, \ldots, N-1$, leads to the final assertion. Watching carefully all the pertinent constants $C$ confirms their independence from $\varepsilon \in(0,1]$.

REMARK 5.3. If we apply Theorem 3.2 and Theorem 5.2 several times, we get rid of the condition that the intervals $I$ and $I_{0}$ contain at most one weight exponent which is not admissible. In this case in the asymptotic representation of the solution $u^{\varepsilon}$ there appear all power solutions to the system (3.14) whose restrictions to $\Omega$ are contained in $\mathscr{D}_{\varkappa, \beta}^{l} V(\Omega ; \varepsilon)$, but not in $\mathscr{D}_{\varkappa, \gamma}^{l} V(\Omega ; \varepsilon)$. The sums in (5.13) (as well as in (3.17)) have to be enlarged to

$$
\sum_{\lambda \in I_{0} \cap \mathbb{Z}} \sum_{j=1}^{J_{\lambda}} b_{\lambda, j} U_{\lambda, j}
$$

where $U_{\lambda, j}=\left(r^{\lambda} \mathcal{V}_{\lambda, j}, r^{\lambda-1} \mathcal{P}_{\lambda, j}\right)$ form a basis for the power solutions to (3.14).

6. Solvability of problem $\left(\mathbf{S}_{\varepsilon}\right)$. Up to now we have no result on the Fredholm property of the operator $A_{\varkappa, \beta}^{l}$ related to problem $\left(\mathrm{S}_{\varepsilon}\right)$. With some technical efforts, Theorem 3.2 can be transmitted to the perturbed problem, this means $A_{\varkappa, \beta}^{l}$ possesses Fredholm property as long as $\beta-\varkappa-1 / 2 \notin \mathbb{Z}$, and corresponding results on the nullity and deficiency also hold true.

However, for our purposes, it is enough to have the result analogous to Proposition 4.3 , i.e. to prove the isomorphism property for $|\beta-\varkappa|<1 / 2$. After removing the inhomogeneity in the boundary conditions, we look for solutions with finite Dirichlet integral, i.e. $\nabla u^{\varepsilon} \in L^{2}(\Omega)$, then use the process of local improvement of smoothness properties and our theorem on asymptotics. To this end we introduce the notion of a weak solution here in the following way. Let $f \in L_{l o c}^{2}(\bar{\Omega})$ be given. We call $u^{\varepsilon} \in L_{l o c}^{2}(\bar{\Omega})^{4}$ with $\nabla u^{\varepsilon} \in L^{2}(\Omega)$ a weak solution to the problem $S_{\varepsilon} u^{\varepsilon}=f, B_{\varepsilon} u^{\varepsilon}=0$, if

$$
\begin{aligned}
b_{\Omega}\left(u^{\varepsilon}, \psi\right):= & \int_{\Omega} \nabla v^{\varepsilon}: \nabla \psi^{\prime}+\varepsilon^{2} \nabla p^{\varepsilon} \nabla \psi_{4} d x \\
& +\int_{\Omega}\left(\nabla \cdot v^{\varepsilon}\right) \psi_{4}-p^{\varepsilon}\left(\nabla \cdot \psi^{\prime}\right) d x=\int_{\Omega} f \cdot \psi d x,
\end{aligned}
$$

for all $\psi=\left(\psi^{\prime}, \psi_{4}\right) \in \mathfrak{C}(\Omega):=C_{0}^{\infty}(\Omega)^{3} \times C_{0}^{\infty}(\bar{\Omega})$, the colon : between two $3 \times 3$ matrices indicates the scalar-product in $\mathbb{R}^{9}$, while the dot $\cdot$ stands for the usual matrix product. If $\Omega$ were bounded, by the Lax Milgram lemma, a weak solution is easily obtained in the Hilbert space $\mathbf{H}(\Omega)=\stackrel{\circ}{H}^{1}(\Omega)^{3} \times H^{1}(\Omega)_{\perp}$ which is the closure of $C_{0}^{\infty}(\Omega)^{3} \times C_{0}^{\infty}(\bar{\Omega})^{\perp}$ with respect to the Dirichlet norm $\left\|\nabla \cdot ; L^{2}(\Omega)\right\|$. As for the exterior domain $\Omega$, the closure of $\mathfrak{C}(\Omega)$ with respect to the Dirichlet norm coincides with $\mathbf{H}(\Omega):=\left\{u=(v, p) \in V_{0}^{1}(\Omega)^{3} \times\right.$ $\left.V_{0}^{1}(\Omega): v_{\mid \partial \Omega}=0\right\}$. But here we come across the difficulties that only the first term in $b_{\Omega}$ is continuous with respect to this norm. Additionally, the functional $\psi \rightarrow \int_{\Omega} f_{4} \cdot \psi_{4} d x$ is not continuous in general. The reduction to the case $f_{4}=0$ will serve both problems. 
Proposition 6.1. For any $\varphi \in L^{2}(\Omega)=V_{0}^{0}(\Omega)$, there exists a vector field $\Phi \in V_{0}^{1}(\Omega)^{3}$ with $\operatorname{div} \Phi=\varphi,\left.\Phi\right|_{\partial \Omega}=0$ and

$$
\left\|\Phi ; V_{0}^{1}(\Omega)\right\| \leq C\left\|\varphi ; V_{0}^{0}(\Omega)\right\|,
$$

with $C$ independent of $\varphi$.

Proof. We use a well known construction. We put $\Phi=\nabla q+\Phi_{l o c}$, where $q \in V_{0}^{2}(\Omega)$ is a solution to the Neumann problem $\Delta q=\varphi$ in $\Omega, \partial_{n} q=0$ on $\partial \Omega$, and $\Phi_{l o c}$ is a local correction. It is well known (see e.g., [6] and [16]) that $q$ exists and is uniquely determined up to a constant, hence

$$
\left\|\nabla q ; V_{0}^{1}(\Omega)\right\| \leq C\left\|\varphi ; V_{0}^{0}(\Omega)\right\| \text {. }
$$

To remove the tangent boundary values of $\nabla q$, we put $\Omega_{\rho}=\Omega \cap B_{\rho}$, where $B_{\rho}$ is an open ball $B_{\rho} \supset \partial \Omega$. We choose $\Phi^{1} \in H^{1}(\Omega)^{3}$ with supp $\Phi^{1} \subset \bar{\Omega}_{\rho}$ such that $\left.\Phi^{1}\right|_{\partial \Omega}=\left.\nabla q\right|_{\partial \Omega}$ and

$$
\left\|\Phi^{1} ; V_{0}^{1}(\Omega)\right\| \leq C\left\|\Phi^{1} ; H_{0}^{1}\left(\Omega_{\rho}\right)\right\| \leq C\left\|\nabla q ; H^{1 / 2}(\partial \Omega)\right\| \leq C\left\|\nabla q ; V_{0}^{1}(\Omega)\right\| .
$$

Of course, $\operatorname{div} \Phi^{1}$ has a support in $\bar{\Omega}_{\rho}$, too, moreover, since $\Phi^{1} \cdot n=0$ on $\partial \Omega_{\rho}$, it holds $\int_{\Omega_{\rho}} \operatorname{div} \Phi^{1}=0$. By the results of [1], e.g., there exists $\Phi^{2} \in H^{1}\left(\Omega_{\rho}\right)^{3}$ with $\operatorname{div} \Phi^{2}=\operatorname{div} \Phi^{1}$ in $\Omega_{\rho}$ and $\Phi^{2}=0$ on $\partial \Omega_{\rho}$, together with the estimate

$$
\left\|\Phi^{2} ; H^{1}\left(\Omega_{\rho}\right)\right\| \leq\left\|\operatorname{div} \Phi^{1} ; L^{2}\left(\Omega_{\rho}\right)\right\| \leq C\left\|\Phi^{1} ; V_{0}^{1}(\Omega)\right\| .
$$

We extend $\Phi^{2}$ by 0 to the whole of $\Omega$ and put $\Phi=\nabla q-\Phi^{1}+\Phi^{2}$, by construction $\operatorname{div} \Phi=\varphi,\left.\Phi\right|_{\partial \Omega}=0$ and collecting (6.3) - (6.5) leads to (6.2).

Theorem 6.2. Let $\varkappa \in[0,3 / 2) \backslash\{1 / 2\}, l \in \mathbb{N}$ with $l-1 \geq \varkappa$, and $\beta \in \mathbb{R}$ such that $|\beta-\varkappa|<$ $1 / 2$. Then the operator $A_{\varkappa, \beta}^{l}$ defines an isomorphism, i.e. for any set of data $(f, g) \in$ $\mathscr{R}_{\varkappa, \beta}^{l} V(\Omega ; \varepsilon)$ (see (4.6)) there exists a unique solution $u^{\varepsilon} \in \mathscr{D}_{\varkappa, \beta}^{l} V(\Omega ; \varepsilon)$ to problem $\left(\mathrm{S}_{\varepsilon}\right)$, and the following estimate holds true with a constant independent of $\varepsilon \in(0,1])$ :

$$
\left\|u^{\varepsilon} ; \mathscr{D}_{\varkappa, \beta}^{l} V(\Omega ; \varepsilon)\right\| \leq C\left\|(f, g) ; \mathscr{R}_{\varkappa, \beta}^{l} V(\Omega ; \varepsilon)\right\| .
$$

Proof. Step 1. Reduction to homogeneous boundary values. By using the result on prolongations of boundary values as mentioned in Section 2 we find $u^{\sharp} \in H^{l+1}(\Omega)^{4}$ with a compact support in $\bar{\Omega}$ such that

$$
\left\|u^{\sharp} ; \mathscr{D}_{\varkappa, \beta}^{l} V(\Omega ; \varepsilon)\right\| \leq C\left\|g ; \mathcal{H}_{\varkappa+1 / 2}^{l+1 / 2}(\partial \Omega)^{3} \times \mathcal{H}_{\varkappa-3 / 2}^{l-1 / 2}(\partial \Omega)\right\|
$$

and $B_{\varepsilon} u^{\sharp}=g$ on $\partial \Omega$. We put $f^{\sharp}=S_{\varepsilon} u^{\sharp}$, it is enough now to find $u^{\varepsilon}$ with $S_{\varepsilon} u^{\varepsilon}=f-f^{\sharp}$ in $\Omega$ and $B_{\varepsilon} u^{\varepsilon}=0$ on $\partial \Omega$, and we keep the inequality

$$
\left\|f^{\sharp} ; V_{\varkappa-1, \beta}^{l-1}(\Omega)^{3} \times V_{\varkappa, \beta}^{l-1}(\Omega)\right\| \leq C\left\|u^{\sharp} ; \mathscr{D}_{\varkappa, \beta}^{l} V(\Omega ; \varepsilon)\right\| \leq\left\|(f, g) ; \mathscr{R}_{\varkappa, \beta}^{l} V(\Omega ; \varepsilon)\right\| .
$$

Step 2. The case $\beta-\varkappa>0$. If $\beta-\varkappa>0$, then $u^{\varepsilon} \in \mathscr{D}_{\varkappa, \beta}^{l} V(\Omega ; \varepsilon)$ (see (4.6)) implies $v^{\varepsilon} \in V^{1}(\Omega), p^{\varepsilon} \in H^{1}(\Omega)$. Now if $u^{\varepsilon}$ solves the homogeneous problem we can use $(6.1)$ with $\psi=u^{\varepsilon}$, since then all integrals converge. Thus $0=b_{\Omega}\left(u^{\varepsilon}, u^{\varepsilon}\right)=\left\|\nabla v^{\varepsilon} ; L^{2}(\Omega)\right\|^{2}+$ $\varepsilon^{2}\left\|\nabla p^{\varepsilon} ; L^{2}(\Omega)\right\|^{2}$, together with $u^{\varepsilon} \in V_{0}^{1}(\Omega)$ this implies $u^{\varepsilon}=0$.

Our next aim is to prove the existence of a weak solution $u^{\varepsilon}$ to the problem $S_{\varepsilon} u^{\varepsilon}=f$ in $\Omega, B_{\varepsilon} u^{\varepsilon}=0$ on $\partial \Omega$ with $(f, 0) \in \mathscr{R}_{\varkappa, \beta}^{l} V(\Omega ; \varepsilon)$. Due to the difficulties mentioned before we cannot directly argue in $\mathbf{H}(\Omega)$. From the definition of $\mathscr{R}_{\varkappa, \beta}^{l} V(\Omega ; \varepsilon)$ we know 
$f_{4} \in V_{\beta-\varkappa}^{0}(\Omega) \subset L^{2}(\Omega)$. Due to Proposition 6.1 there exists $v^{1} \in V_{0}^{1}(\Omega)^{3}$ with vanishing boundary values, $\operatorname{div} v^{1}=f_{4}$ and

$$
\left\|v^{1} ; V_{0}^{1}(\Omega)\right\| \leq C\left\|f_{4} ; V_{0}^{0}(\Omega)\right\| \leq C\left\|f_{4} ; V_{\beta}^{\varkappa}(\Omega)\right\| .
$$

We search for $u^{\varepsilon}=\left(v^{1}, 0\right)+\mathfrak{u}^{\varepsilon}$, then it suffices to find $\mathfrak{u}^{\varepsilon}$ with

$$
b_{\Omega}\left(\mathfrak{u}^{\varepsilon} ; \psi\right)=\int_{\Omega}\left(f^{\prime} \cdot \psi^{\prime}-\nabla v^{1} \nabla \psi^{\prime}\right) d x
$$

for all $\psi \in \mathfrak{C}(\Omega)$. We fix $\rho$ as in the proof of Proposition 6.1 , for $R>\rho$, we put $\Omega_{R}=$ $\{x \in \Omega:|x|<R\}$. To $\Omega_{R}$ we assign the space

$$
\mathfrak{C}\left(\Omega_{R}\right)=\left\{\psi=\left(\psi^{\prime}, \psi_{4}\right) \in C^{\infty}(\bar{\Omega})^{4}: \operatorname{supp} \psi^{\prime} \subset \Omega_{R}, \psi_{4}=0 \text { for }|x|=R\right\}
$$

and the Hilbert space $\mathbf{H}\left(\Omega_{R}\right)$ as the closure of $\mathfrak{C}\left(\Omega_{R}\right)$ with respect to the Dirichlet norm on $\Omega_{R}$. Thanks to Hardy's inequality, we have

$$
\left\|r^{-1} \psi ; L^{2}\left(\Omega_{R}\right)\right\| \leq C\left\|\nabla \psi ; L^{2}\left(\Omega_{R}\right)\right\| \text { for all } \psi \in \mathfrak{C}\left(\Omega_{R}\right)
$$

with a constant independent of $R$ and $\psi$. For $f^{\prime} \in V_{\varkappa-1, \beta}^{l-1}(\Omega ; \varepsilon)^{3}$, we have

$$
\left\|f^{\prime} ; V_{\beta}^{\varkappa-1}(\Omega)\right\| \leq\left\|f^{\prime} ; V_{\varkappa-1, \beta}^{l-1}(\Omega ; \varepsilon)\right\| .
$$

If $\varkappa \geq 1$, it follows $V_{\beta}^{\varkappa-1}(\Omega) \subset V_{\beta-\varkappa+1}^{0}(\Omega) \subset V_{1}^{0}(\Omega)$. If $\varkappa \in[0,1)$, then according to (3.2), $f_{i}=f_{i}^{0}+\sum_{j} \partial_{j} F_{i j}, i=1,2,3$ with

$$
\left\|f^{\prime} ; V_{\beta}^{\varkappa-1}(\Omega)\right\| \leq\left\|f^{0} ; V_{\beta-\varkappa+1}^{0}(\Omega)\right\|+\sum_{i, j}\left\|F_{i j} ; V_{\beta}^{\varkappa}(\Omega)\right\| \leq 2\left\|f^{\prime} ; V_{\beta}^{\varkappa-1}(\Omega)\right\| .
$$

Since $\beta \geq \varkappa$, we have $V_{\beta}^{\varkappa}(\Omega) \subset L^{2}(\Omega)$, in both cases we end with the following estimate (with $F_{i j}=0$ for $\varkappa \geq 1$ )

$$
\begin{aligned}
& \left|\int_{\Omega}\left(f^{\prime} \cdot \psi^{\prime}-\nabla v^{1}: \nabla \psi^{1}\right) d x\right| \leq\left\|r f^{0} ; L^{2}\left(\Omega_{R}\right)\right\|\left\|r^{-1} \psi^{\prime} ; L^{2}\left(\Omega_{R}\right)\right\| \\
& \quad+\left(\sum_{i, j}\left\|F_{i j} ; L^{2}\left(\Omega_{R}\right)\right\|+\left\|\nabla v^{1} ; L^{2}\left(\Omega_{R}\right)\right\|\right)\left\|\nabla \psi^{\prime} ; L^{2}\left(\Omega_{R}\right)\right\| \\
& \leq C\left(\left\|f^{0} ; V_{1}^{0}(\Omega)\right\|+\sum_{i, j}\left\|F_{i, j} ; V_{0}^{0}(\Omega)\right\|+\left\|f_{4} ; V_{0}^{0}(\Omega)\right\|\right)\left\|\nabla \psi^{\prime} ; L^{2}\left(\Omega_{R}\right)\right\| \\
& \leq 3 C\left\|(f, 0) ; \mathscr{R}_{\varkappa, \beta}^{l} V(\Omega ; \varepsilon)\right\|\left\|\nabla \psi^{\prime} ; \Omega_{R}\right\|
\end{aligned}
$$

with a constant $C$ independent of $f, R$ and $\varepsilon \in(0,1]$. The bilinear form $b_{\Omega_{R}}$ is continuous and coercive on $\mathbf{H}\left(\Omega_{R}\right)$ for any $R>\rho$, thus, the Lax-Milgram lemma provides us with a unique solution $\mathfrak{u}_{R}^{\varepsilon} \in \mathbf{H}\left(\Omega_{R}\right)$ fulfilling

$$
b_{\Omega_{R}}\left(\mathfrak{u}_{R}^{\varepsilon} ; \psi\right)=\int_{\Omega_{R}}\left(f^{\prime} \cdot \psi^{\prime}-\nabla v^{1}: \nabla \psi^{\prime}\right) d x
$$

for any $\psi \in \mathbf{H}\left(\Omega_{R}\right)$. Because

$$
b_{\Omega_{R}}\left(\mathfrak{u}_{R}^{\varepsilon} ; \mathfrak{u}_{R}^{\varepsilon}\right)=\left\|\nabla \mathfrak{v}_{R}^{\varepsilon} ; L^{2}\left(\Omega_{R}\right)\right\|^{2}+\varepsilon^{2}\left\|\nabla \mathfrak{p}_{R}^{\varepsilon}\right\|^{2},
$$

the a priori inequality

$$
\begin{aligned}
& \left\|r^{-1} \mathfrak{v}_{R}^{\varepsilon} ; L^{2}\left(\Omega_{R}\right)\right\|+\left\|\nabla \mathfrak{v}_{R}^{\varepsilon} ; L^{2}\left(\Omega_{R}\right)\right\| \\
& \quad+\quad \varepsilon\left(\left\|r^{-1} \mathfrak{p}_{R}^{\varepsilon} ; L^{2}\left(\Omega_{R}\right)\right\|+\left\|\nabla \mathfrak{p}_{R}^{\varepsilon} ; L^{2}\left(\Omega_{R}\right)\right\|\right) \leq C\left\|(f, 0) ; \mathscr{R}_{\varkappa, \beta}^{l} V(\Omega ; \varepsilon)\right\|
\end{aligned}
$$


is valid independent of $R>\rho$ and $\varepsilon \in(0,1]$, thanks to (6.10) and (6.11). Extending $\mathfrak{u}_{R}^{\varepsilon}$ by 0 to the whole domain $\Omega$, this gives

$$
\left\|\mathfrak{v}_{R}^{\varepsilon} ; V_{0}^{1}(\Omega)\right\|+\varepsilon\left\|\mathfrak{p}_{R}^{\varepsilon} ; V_{0}^{1}(\Omega)\right\| \leq C\left\|(f, 0) ; \mathscr{R}_{\varkappa, \beta}^{l} V(\Omega ; \varepsilon)\right\| .
$$

Thus, there exists a sequence $R_{n}$ with $R_{n} \rightarrow \infty$ such that $\mathfrak{u}_{R_{n}}^{\varepsilon}$ converges weakly in $V_{0}^{1}(\Omega)^{4}$ to a solution $\mathfrak{u}^{\varepsilon}$ of (6.9), and (6.12) is preserved for $\mathfrak{u}^{\varepsilon}$. Clearly, $\mathfrak{u}^{\varepsilon}=\left(v^{1}, 0\right)+\mathfrak{u}^{\varepsilon}$ fulfils (6.1) and the inequality

$$
\left\|v^{\varepsilon} ; V_{0}^{1}(\Omega)\right\|+\varepsilon\left\|p^{\varepsilon} ; V_{0}^{1}(\Omega)\right\| \leq C\left\|(f, 0) ; \mathscr{R}_{\varkappa, \beta}^{l} V(\Omega ; \varepsilon)\right\| .
$$

Since we finally want $u^{\varepsilon} \in \mathscr{D}_{\varkappa, \beta}^{l} V(\Omega ; \varepsilon)$, we need to improve the regularity and decay properties. Since $f \in H_{l o c}^{l-1}(\bar{\Omega})$, we clearly obtain $u^{\varepsilon} \in H_{l o c}^{l+1}(\bar{\Omega})$. As for decay properties, in particular of $p^{\varepsilon}$, the estimate (6.13) is too weak - with respect to $\varepsilon$ and $\beta$. In order to remove this, we employ Theorem 5.2. We have $v^{\varepsilon} \in V^{1}(\Omega) \subset V_{-1}^{0}(\Omega) \subset V_{-2}^{0}(\Omega)$, while $p^{\varepsilon} \in V_{-1}^{0}(\Omega)$. Since $\mathscr{R}_{\varkappa, \beta}^{l} V(\Omega ; \varepsilon) \subset \mathscr{R}_{\varkappa, \tilde{\beta}}^{l}(\Omega ; \varepsilon)$ for $\beta \geq \tilde{\beta}$, for $\beta>\varkappa$ we get

$$
\left\|(f, 0) ; \mathscr{R}_{\varkappa, \beta}^{l} V(\Omega ; \varepsilon)\right\| \leq C\left\|(f, 0) ; \mathscr{R}_{\varkappa, \varkappa-1}^{l}(\Omega ; \varepsilon)\right\|
$$

with $C$ independent of $\varepsilon$. Lemma 4.2 leads to

$$
\left\|u^{\varepsilon} ; \mathscr{D}_{\varkappa, \varkappa-1}^{l}(\Omega ; \varepsilon)\right\| \leq C\left(\left\|(f, 0) ; \mathscr{R}_{\varkappa, \varkappa-1}^{l}(\Omega ; \varepsilon)\right\|+\left\|v^{\varepsilon} ; V_{-2}^{0}(\Omega)\right\|+\left\|p^{\varepsilon} ; V_{-1}^{0}(\Omega)\right\|\right) .
$$

Theorem 5.2 on asymptotics, here used for the weight indices $\beta, \varkappa-1$, implies the following asymptotic representation (observe the restriction $\varkappa+1 / 2>\beta>\varkappa \geq 0$ )

$$
u^{\varepsilon}=\sum_{j=1}^{3} b_{j} \mathrm{e}_{j}+\tilde{u}^{\varepsilon}
$$

where $\mathrm{e}_{j}$ the $j$-th unit vector in $\mathbb{R}^{4}$, moreover,

$$
\begin{aligned}
& \sum_{j=1}^{3}\left|b_{j}\right|+\left\|\tilde{u}^{\varepsilon} ; \mathscr{R}_{\varkappa, \beta}^{l} V(\Omega ; \varepsilon)\right\| \\
& \quad \leq C\left(\left\|(f, 0) ; \mathscr{R}_{\varkappa, \beta}^{l} V(\Omega ; \varepsilon)\right\|+\left\|u^{\varepsilon} ; \mathscr{D}_{\varkappa, \varkappa-1}^{l}(\Omega ; \varepsilon)\right\|\right) \\
& \leq C\left(\left\|(f, 0) ; \mathscr{R}_{\varkappa, \beta}^{l} V(\Omega ; \varepsilon)\right\|+\left\|v^{\varepsilon} ; V_{-2}^{0}(\Omega)\right\|+\left\|p^{\varepsilon} ; V_{-1}^{0}(\Omega ; \varepsilon)\right\|\right),
\end{aligned}
$$

where for the second inequality we used (6.14) and (6.15). Since we know already $u^{\varepsilon} \in$ $V_{-1}^{0}(\Omega)$, which can only be true if all the three constants $b_{j}$ vanish, thus $u^{\varepsilon} \in \mathscr{D}_{\varkappa, \beta}^{l} V(\Omega ; \varepsilon)$. From (6.13) we get

$$
\left\|v^{\varepsilon} ; V_{-2}^{0}(\Omega)\right\| \leq C\left\|v^{\varepsilon} ; V_{-1}^{0}(\Omega)\right\| \leq C\left\|(f, 0) ; \mathscr{R}_{\varkappa, \beta}^{l} V(\Omega ; \varepsilon)\right\|,
$$

and it remains to find a suitable estimate for $p^{\varepsilon}$ in order to remove the second term in (6.16). Since $u^{\varepsilon} \in \mathscr{D}_{\varkappa, \beta}^{l} V(\Omega ; \varepsilon) \subset \mathscr{D}_{\beta}^{\varkappa} V(\Omega)$, we know already $p^{\varepsilon} \in V_{\beta}^{\varkappa}(\Omega) \subset V_{0}^{0}(\Omega)$ and we need only to control the latter norm itself. Let $\varphi \in V_{0}^{0}(\Omega)$ be arbitrary and $\Phi \in V_{0}^{1}(\Omega)$ the solution to $\operatorname{div} \Phi=\varphi$ according to Proposition 6.1. Furthermore, we choose a sequence $\Phi_{n} \subset C_{0}^{\infty}(\Omega)^{3}$ converging to $\Phi$ in $V_{0}^{1}(\Omega)$. We use $(6.1)$ for $\psi=\left(\Phi_{n}, 0\right)$ and find

$$
\int_{\Omega} \nabla v^{\varepsilon}: \nabla \Phi_{n} d x-\int_{\Omega} f^{\prime} \cdot \Phi_{n} d x=\int_{\Omega} p^{\varepsilon} \operatorname{div} \Phi_{n} d x .
$$


Here it is possible to pass to the limit in all terms, and repeating the arguments for (6.11) leads to

$$
\begin{aligned}
& \left|\int_{\Omega} p^{\varepsilon} \varphi d x\right|=\left|\int_{\Omega}\left(\nabla v^{\varepsilon}: \nabla \Phi-f^{\prime} \Phi\right) d x\right| \\
& \leq C\left\|(f, 0) ; \mathscr{R}_{\varkappa, \beta}^{l} V(\Omega ; \varepsilon)\right\|\left\|\Phi ; V_{0}^{1}(\Omega)\right\| \\
& \leq C\left\|(f, 0) ; \mathscr{R}_{\varkappa, \beta}^{l} V(\Omega ; \varepsilon)\right\|\left\|\varphi ; V_{0}^{0}(\Omega)\right\|,
\end{aligned}
$$

which leads to

$$
\left\|p^{\varepsilon}, V_{0}^{0}(\Omega)\right\| \leq C\left\|(f, 0) ; \mathscr{R}_{\varkappa, \beta}^{l} V(\Omega ; \varepsilon)\right\|
$$

independent of $\varepsilon \in(0,1]$. Finally, we have proved that the right-hand side of $(6.16)$ is majorized by $C\left\|(f, 0) ; \mathscr{R}_{\varkappa, \beta}^{l} V(\Omega ; \varepsilon)\right\|$.

Step 3. The case $\beta-\varkappa \leq 0$. We now fix $\varkappa \in[0,3 / 2) \backslash\{1 / 2\}$, and $\beta \in \mathbb{R}$ with $\beta-\varkappa \in$ $(-1 / 2,0)$. It is enough to extend (6.6) to this case, too. Since, by Step 3, we already know the existence of solutions in $\mathscr{D}_{\varkappa, \beta}^{l} V(\Omega ; \varepsilon)$ for data with compact support in $\bar{\Omega}$, the result then follows from density arguments. Furthermore, by Step 1, we may restrict ourselves to the case $B_{\varepsilon} u^{\varepsilon}=0$ and start with the uniqueness of solutions. If $u^{\varepsilon} \in \mathscr{D}_{\varkappa, \beta}^{l} V(\Omega ; \varepsilon)$ is a solution to the homogeneous problem, then Theorem 5.2 gives $u^{\varepsilon} \in \mathscr{D}_{\varkappa, \tilde{\beta}}^{l} V(\Omega ; \varepsilon)$ for any $\tilde{\beta} \in[\varkappa, \varkappa+1 / 2)$ (observe that the interval $(-1 / 2,1 / 2)$ is free of critical values for $\beta-\varkappa$ ) hence $u^{\varepsilon}=0$ by Step 2. To see (6.6) we apply a modification of standard compactness arguments: We choose $\delta>0$ small enough such that still $\beta-\delta-\varkappa \in(-1 / 2,0)$ is valid. For $u \in \mathscr{D}_{\varkappa, \beta}^{l} V(\Omega ; \varepsilon)$ with $B_{\varepsilon} u=0, S_{\varepsilon} u=f$, from Theorem 5.2 and Lemma 4.2 it follows

$$
\begin{aligned}
\| u ; & \mathscr{D}_{\varkappa, \beta}^{l} V(\Omega ; \varepsilon) \| \leq C\left(\left\|(f, 0) ; \mathscr{R}_{\varkappa, \beta}^{l} V(\Omega ; \varepsilon)\right\|+\left\|u ; \mathscr{D}_{\varkappa, \beta-\delta}^{l} V(\Omega ; \varepsilon)\right\|\right) \\
\leq & C\left(\left\|(f, 0) ; \mathscr{R}_{\varkappa, \beta}^{l} V(\Omega ; \varepsilon)\right\|+\left\|(f, 0) ; \mathscr{R}_{\varkappa, \beta-\delta}^{l} V(\Omega ; \varepsilon)\right\|\right. \\
& \left.+\left\|v ; V_{\beta-\delta-\varkappa-1}^{0}(\Omega)\right\|+\left\|p ; V_{\beta-\delta-\varkappa}^{0}(\Omega)\right\|\right) \\
\leq & C\left(\left\|(f, 0) ; \mathscr{R}_{\varkappa, \beta}^{l} V(\Omega ; \varepsilon)\right\|+\left\|v ; V_{\beta-\delta-\varkappa-1}^{0}(\Omega)\right\|+\left\|p ; V_{\beta-\delta-\varkappa}^{0}(\Omega)\right\|\right) .
\end{aligned}
$$

The last two terms have to be removed. To this end, we recall the embeddings

$$
\mathscr{D}_{\varkappa, \beta}^{l} V(\Omega ; \varepsilon) \subset V_{\beta}^{\varkappa+1}(\Omega)^{3} \times V_{\beta}^{\varkappa}(\Omega) \subset V_{\varkappa-\beta-\delta-1}^{0}(\Omega)^{3} \times V_{\varkappa-\beta-\delta}^{0}(\Omega)=: \mathbf{V}^{0} .
$$

The embedding constants are bounded independent of $\varepsilon$ and the second embedding is compact. Assume (6.6) is not true, then there exist sequences $\left\{\varepsilon_{n}\right\}_{n \in \mathbb{N}} \subset(0,1]$ and $\left\{u_{n}\right\} \subset \mathscr{D}_{\varkappa, \beta}^{l} V(\Omega ; \varepsilon)$ with $\left\|u_{n} ; \mathbf{V}^{0}\right\|=1$ and $\left\|\left(S_{\varepsilon_{n}} u_{n}, 0\right) ; \mathscr{R}_{\varkappa, \beta}^{l} V\left(\Omega ; \varepsilon_{n}\right)\right\| \rightarrow 0$ as $n \rightarrow \infty$. Without loss of generality we require $\lim _{n \rightarrow \infty} \varepsilon_{n}=\varepsilon_{0}$. Then (6.17) and (6.18) imply that $\left\|u_{n}, \mathscr{D}_{\beta}^{\varkappa} V(\Omega)\right\|$ remains bounded. After possibly extracting a subsequence, we obtain that $u_{n}$ weakly converges to $u_{0} \in \mathscr{D}_{\beta}^{\varkappa} V(\Omega)$. Since $\left\|\cdot ; \mathscr{R}_{\beta}^{\varkappa} V(\Omega)\right\| \leq\left\|\cdot ; \mathscr{R}_{\varkappa, \beta}^{l} V(\Omega ; \varepsilon)\right\|$ for all $\varepsilon \in(0,1]$, we know that

$$
\begin{aligned}
-\Delta v_{n}+\nabla p_{n} & \rightarrow 0 \text { in } V_{\beta}^{\varkappa-1}(\Omega), \\
\operatorname{div} v_{n}-\varepsilon_{n}^{2} \Delta p_{n} & \rightarrow 0 \text { in } V_{\beta}^{\varkappa}(\Omega) .
\end{aligned}
$$

(6.19) gives already $-\Delta v_{0}+\nabla p_{0}=0$. As for the limits in (6.20) we convince us first that $\varepsilon_{0}>0$. On one hand, as $V_{\beta}^{\varkappa}(\Omega) \subset V_{\beta-2}^{\varkappa-2}(\Omega)$, it follows $\left\|\operatorname{div} v_{n}-\varepsilon_{n}^{2} \Delta p_{n} ; V_{\beta-2}^{\varkappa-2}(\Omega)\right\| \rightarrow 0$. 
On the other hand,

$$
\left\|\Delta p_{n} ; V_{\beta-2}^{\varkappa-2}(\Omega)\right\| \leq C\left\|\Delta p_{n} ; V_{\beta}^{\varkappa-2}(\Omega)\right\| \leq C\left\|p_{n} ; V_{\beta}^{\varkappa}(\Omega)\right\| \leq \tilde{C}
$$

which implies $\operatorname{div} v_{n} \rightarrow 0$ in $V_{\beta-2}^{\varkappa-2}(\Omega)$ if $\varepsilon_{0}=0$. Since div $v_{n}$ converges to div $v_{0}$ weakly in $V_{\beta}^{\varkappa}(\Omega)$, this leads to $\operatorname{div} v_{0}=0$. Thus $u_{0} \in \mathscr{D}_{\beta}^{\varkappa} V(\Omega)$ solves the homogeneous Stokes problem, and Theorem 3.2 leads to $u^{0}=0$, which contradicts $\left\|u_{0} ; \mathbf{V}^{0}\right\|=1$ (recall $u_{n}$ converges to $u_{0}$ strongly in $\mathbf{V}^{0}$ ). Thus, $\varepsilon_{0}>0$ must hold. But then, for $\varepsilon_{0} / 2 \leq \varepsilon \leq 1$, all $V_{\varkappa, \beta}^{l}(\Omega ; \varepsilon)$-norms are equivalent to $\left\|\cdot ; V_{\varkappa, \beta}^{l}(\Omega ; 1)\right\|$ independent of $\varepsilon$. Hence $u_{n}$ converges weakly in $\mathscr{D}_{\varkappa, \beta}^{l} V(\Omega ; 1)$ to a solution $u_{0}$ of $S_{\varepsilon_{0}} u^{0}=0, B_{\varepsilon_{0}} u^{0}=0$, which leads to the same contradiction, since we know already the uniqueness result.

7. Weighted spaces with detached asymptotics and error estimates. Proposition 4.3 and Theorem 6.2 provide us with a common frame for the solutions to the problems $\left(\mathrm{S}_{0}\right)$ and $\left(\mathrm{S}_{\varepsilon}\right)$. In order to take into account the behavior at infinity of the solution while comparing $u^{0}$ and $u^{\varepsilon}$, we introduce spaces with detached asymptotics. Let $\varkappa \in[0,3 / 2) \backslash\{1 / 2\}, l \in \mathbb{N}$ with $l-1 \geq \varkappa, \gamma \in \mathbb{R}$ with

$$
\gamma-\varkappa \in(m-1 / 2, m+1 / 2), \quad m \in \mathbb{N}_{0} \text { fixed. }
$$

For $\left(f, g^{\prime}\right) \in \mathscr{R}_{\gamma}^{\varkappa} V(\Omega) \subset \mathscr{R}_{\gamma-m}^{\varkappa} V(\Omega)$, by Theorem 3.2 , there exists a unique solution $u^{0} \in \mathscr{D}_{\gamma-m}^{\varkappa} V(\Omega)$ to problem $\left(\mathrm{S}_{0}\right)$. Let $U^{1}, \ldots, U^{N}$ be a basis for the power solutions of the form (3.13) with $\lambda=1, \ldots, m-1$, whose restrictions to $\Omega$ are contained in $\mathscr{D}_{\gamma-m}^{\varkappa} V(\Omega)$, but not in $\mathscr{D}_{\gamma}^{\varkappa} V(\Omega)$. Then, by (3.18), we obtain the representation

$$
u^{0}=\sum_{j=1}^{N} b_{q}^{0} U^{q}+\tilde{u}^{0}, \quad \tilde{u}^{0} \in \mathscr{D}_{\gamma}^{\varkappa} V(\Omega),
$$

with now uniquely determined coefficients $b_{q}^{0}$ and a uniquely determined remainder $\tilde{u}^{0}$, together with the estimate

$$
\left\|\tilde{u}^{0} ; \mathscr{D}_{\gamma}^{\varkappa} V(\Omega)\right\|+\sum_{j=1}^{N}\left|b_{q}^{0}\right| \leq C\left\|\left(f, g^{\prime}\right) ; \mathscr{R}_{\gamma}^{\varkappa} V(\Omega)\right\| .
$$

We extend this result to the solutions of $\left(\mathrm{S}_{0}\right)$ and $\left(\mathrm{S}_{\varepsilon}\right)$ in step weighted spaces.

Proposition 7.1. Let $\varkappa, l, m$ and $\gamma$ fulfil (7.1). For any $\left(f, g^{\prime}\right) \in \mathbf{R}_{\varkappa, \gamma}^{l} V(\Omega ; \varepsilon)$ (compare (4.9)) the solution $u^{0} \in \mathbf{D}_{\varkappa, \gamma-m}^{l} V(\Omega ; \varepsilon)$ to problem $\left(\mathrm{S}_{0}\right)$ possesses the uniquely determined representation (7.2) with $\tilde{u}^{0} \in \mathbf{D}_{\varkappa, \gamma}^{\varkappa} V(\Omega ; \varepsilon)$, and the following estimate is valid:

$$
\sum_{j=1}^{N}\left|b_{q}^{0}\right|+\left\|\tilde{u}^{0} ; \mathbf{D}_{\varkappa, \gamma}^{l} V(\Omega ; \varepsilon)\right\| \leq C\left\|\left(f, g^{\prime}\right) ; \mathbf{R}_{\varkappa, \gamma}^{l} V(\Omega ; \varepsilon)\right\| .
$$

For $(f, g) \in \mathscr{R}_{\varkappa, \gamma}^{l} V(\Omega ; \varepsilon)$ the solution $u^{\varepsilon} \in \mathscr{D}_{\varkappa, \gamma-m}^{l} V(\Omega ; \varepsilon)$ to problem $\left(\mathrm{S}_{\varepsilon}\right)$ admits the uniquely determined representation

$$
u^{\varepsilon}=\sum_{q=0}^{N} b_{q}^{\varepsilon} U^{q}+\tilde{u}^{\varepsilon}
$$


and

$$
\sum_{j=1}^{N}\left|b_{q}^{\varepsilon}\right|+\left\|\tilde{u}^{\varepsilon} ; \mathscr{D}_{\varkappa, \gamma}^{l} V(\Omega ; \varepsilon)\right\| \leq C\left\|(f, g) ; \mathscr{R}_{\varkappa, \gamma}^{l} V(\Omega ; \varepsilon)\right\| .
$$

In both inequalities the constants are independent of $\varepsilon \in(0,1]$.

Proof. Like in Kondratiev spaces we have $\mathbf{R}_{\varkappa, \gamma}^{l} V(\Omega ; \varepsilon) \subset \mathbf{R}_{\varkappa, \gamma-m}^{l} V(\Omega ; \varepsilon)$, thus by Proposition 4.3 we obtain a unique solution $u^{0} \in \mathbf{D}_{\varkappa, \gamma-m}^{l} V(\Omega ; \varepsilon)$, together with the $\varepsilon$-independent estimate

$$
\left\|u^{0} \in \mathbf{D}_{\varkappa, \gamma-m}^{l} V(\Omega ; \varepsilon)\right\| \leq C\left\|(f, g) ; \mathbf{R}_{\varkappa, \gamma-m}^{l} V(\Omega ; \varepsilon)\right\| \leq C\left\|(f, g) ; \mathbf{R}_{\varkappa, \gamma}^{l} V(\Omega ; \varepsilon)\right\| .
$$

Since $\mathbf{R}_{\varkappa, \beta}^{l} V(\Omega ; \varepsilon) \subset \mathscr{R}_{\beta}^{\varkappa}(\Omega)$, and $\mathbf{D}_{\varkappa, \beta}^{l} V(\Omega ; \varepsilon) \subset \mathscr{D}_{\beta}^{\varkappa} V(\Omega)$ for any $\beta \in \mathbb{R}$, we have already (7.2) together with (7.3). The estimates for the higher order derivatives follow then by repeating the arguments of Proposition 4.3. The assertion for $u^{\varepsilon}$ follows from Theorem 6.2, Theorem 5.2, Remark 5.3 and (6.6) applied to $\beta=\gamma-m$.

We can reformulate these result in terms of spaces with detached asymptotics. We put

$$
\mathfrak{D}_{\varkappa, \gamma}^{l, 0} V(\Omega ; \varepsilon)=\{u: u \text { fulfils }(7.2)\},
$$

while the norm in $\mathfrak{D}_{\varkappa, \gamma}^{l, 0} V(\Omega ; \varepsilon)$ is defined by the left-hand side of (7.4). Then $\mathfrak{D}_{\varkappa, \gamma}^{l, 0} V(\Omega ; \varepsilon)$ is isomorphic to $\mathbb{R}^{N} \times \mathbf{D}_{\varkappa, \gamma}^{l} V(\Omega ; \varepsilon)$. In a similar way we introduce $\mathfrak{D}_{\varkappa, \gamma}^{l, \varepsilon} V(\Omega ; \varepsilon)$, isomorphic to $\mathbb{R}^{N} \times \mathscr{D}_{\varkappa, \gamma}^{l} V(\Omega ; \varepsilon)$, as the space of functions fulfilling $(7.5)$, provided with the norm defined by the left-hand side of (7.6). Then

$$
\left(S_{0}, B_{0}\right): \mathfrak{D}_{\varkappa, \gamma}^{l, 0} V(\Omega ; \varepsilon) \rightarrow \mathbf{R}_{\varkappa, \gamma}^{l} V(\Omega ; \varepsilon), \quad\left(S_{\varepsilon}, B_{\varepsilon}\right): \mathfrak{D}_{\varkappa, \gamma}^{l, \varepsilon} V(\Omega ; \varepsilon) \rightarrow \mathscr{R}_{\varkappa, \gamma}^{l} V(\Omega ; \varepsilon)
$$

define isomorphisms. Now let $\left(f, g^{\prime}\right) \in \mathbf{R}_{\varkappa, \gamma}^{l} V(\Omega ; \varepsilon)$ be fixed and $u^{0} \in \mathfrak{D}_{\varkappa, \gamma}^{l, 0} V(\Omega ; \varepsilon)$ be the solution to $\left(S_{0}\right)$. We put $g_{4}=0$, then $(f, g) \in \mathscr{R}_{\varkappa, \gamma}^{l} V(\Omega ; \varepsilon)$. Let $u^{\varepsilon} \in \mathfrak{D}_{\varkappa, \gamma}^{l, \varepsilon} V(\Omega ; \varepsilon)$ be the unique solution to $\left(\mathrm{S}_{\varepsilon}\right)$. Clearly $u^{\varepsilon} \in \mathfrak{D}_{\varkappa, \gamma}^{l, 0} V(\Omega ; \varepsilon)$, too. Moreover, since $U_{4}^{j}=P^{j}$ is harmonic for all $j=1, \ldots, N$, we have $\Delta p^{\varepsilon}=\Delta \tilde{p}^{\varepsilon}$. Thus the difference $u^{0}-u^{\varepsilon}$ solves the problem $\left(\mathrm{S}_{0}\right)$ with right-hand side

$$
f^{\prime}=0, \quad f_{4}=\varepsilon^{2} \Delta \tilde{p}^{\varepsilon}, \quad g^{\prime}=0 .
$$

We apply Lemma 5.1 to find the final error estimate.

TheOREM 7.2. Let $\varkappa, l, m$ and $\gamma$ fulfill (7.1). For $\left(f, g^{\prime}\right) \in \mathbf{R}_{\varkappa, \gamma}^{l} V(\Omega ; \varepsilon)$ (see (4.10)) let $u^{0} \in \mathfrak{D}_{\varkappa, \gamma}^{l, 0} V(\Omega ; \varepsilon) \subset \mathbf{D}_{\varkappa, \gamma-m}^{l} V(\Omega ; \varepsilon)$ (see (4.9)) be the unique solution to the exterior Stokes problem and $u^{\varepsilon} \in \mathfrak{D}_{\varkappa, \gamma}^{l, \varepsilon} V(\Omega ; \varepsilon) \subset \mathscr{D}_{\varkappa, \gamma-m}^{l} V(\Omega ; \varepsilon)$ be the unique solution to the perturbed problem $\left(\mathrm{S}_{\varepsilon}\right)$ with $\left(g=g^{\prime}, 0\right)$. Then the difference $u^{0}-u^{\varepsilon}$ fulfills the following error estimate

$$
\begin{aligned}
& \sum_{j=1}^{N}\left|b_{j}^{0}-b_{j}^{\varepsilon}\right|+\left\|\tilde{u}^{0}-\tilde{u}^{\varepsilon} ; \mathscr{D}_{0, \beta-\varkappa}^{l-1} V(\Omega ; \varepsilon)\right\| \\
& =\sum_{j=1}^{N}\left|b_{j}^{0}-b_{j}^{\varepsilon}\right|+\left\|\tilde{v}^{0}-\tilde{v}^{\varepsilon} ; V_{1, \beta-\varkappa}^{l} V(\Omega ; \varepsilon)\right\|+\left\|\tilde{p}^{0}-\tilde{p}^{\varepsilon} ; V_{0, \beta-\varkappa}^{l-1}(\Omega ; \varepsilon)\right\| \\
& \leq C \varepsilon^{\varkappa}\left\|\left(f, g^{\prime}\right) ; \mathbf{R}_{\varkappa, \beta}^{l} V(\Omega ; \varepsilon)\right\|
\end{aligned}
$$

with a constant $C$ independent of $\varepsilon \in(0,1]$. 
Proof. We have $u^{0}, u^{\varepsilon} \in \mathfrak{D}_{\varkappa, \gamma}^{l, 0} V(\Omega ; \varepsilon)$ and $u^{0}-u^{\varepsilon}$ solves $\left(\mathrm{S}_{0}\right)$ with data (7.7). Since $\varkappa \geq 0$, the estimate (5.8) in Lemma 5.1, together with (7.6) implies

$$
\begin{aligned}
& \left\|\varepsilon^{2} \Delta \tilde{p}^{\varepsilon} ; V_{0, \gamma-\varkappa}^{l-1}(\Omega ; \varepsilon)\right\| \leq C\left\|\varepsilon^{2} \Delta \tilde{p}^{\varepsilon} ; V_{0, \gamma}^{l-1}(\Omega ; \varepsilon)\right\| \leq C \varepsilon^{\varkappa}\left\|\tilde{p}^{\varepsilon} ; V_{\varkappa, \gamma}^{l+1}(\Omega ; \varepsilon)\right\| \\
& \leq C \varepsilon^{\varkappa}\left\|\left(f,\left(g^{\prime}, 0\right)\right) ; \mathscr{R}_{\varkappa, \gamma}^{l} V(\Omega ; \varepsilon)\right\| \leq C \varepsilon^{\varkappa}\left\|\left(f, g^{\prime}\right) ; \mathbf{R}_{\varkappa, \gamma}^{l} V(\Omega ; \varepsilon)\right\| .
\end{aligned}
$$

We want to employ (7.4) on $u^{0}-u^{\varepsilon}$, but we have to reduce the order of the estimated derivatives, since by the previous inequality we can only control $\left\|\tilde{u}^{0}-\tilde{u}^{\varepsilon} ; \mathfrak{D}_{0, \gamma-\varkappa}^{l-1} V(\Omega ; \varepsilon)\right\|$. Finally we arrive at

$$
\sum_{j=1}^{N}\left|b_{j}^{0}-b_{j}^{\varepsilon}\right|+\left\|\tilde{u}^{0}-\tilde{u}^{\varepsilon} ; \mathbf{D}_{0, \gamma-\varkappa}^{l-1} V(\Omega ; \varepsilon)\right\| \leq C\left\|\varepsilon^{2} \Delta \tilde{p}^{\varepsilon} ; V_{0, \gamma-\varkappa}^{l-1}(\Omega ; \varepsilon)\right\|,
$$

which leads to $(7.8)$ by $(7.9)$.

REMARK 7.3. Let us add some comments. Like in [10], it can be shown that these estimates are asymptotically precise with respect to $\varepsilon$. Of course, with $\varepsilon \searrow 0$, only the stable part of the left-hand side is relevant for the order of convergence. This means for $l \geq \max \{\varkappa+1,2\}$

$$
\sum_{j=1}^{N}\left|b_{j}^{0}-b_{j}^{\varepsilon}\right|+\left\|\tilde{v}^{0}-\tilde{v}^{\varepsilon} ; V_{\gamma-\varkappa}^{1}(\Omega)\right\|+\left\|\tilde{p}^{0}-\tilde{p}^{\varepsilon} ; V_{\gamma-\varkappa}^{0}(\Omega)\right\|=O\left(\varepsilon^{\varkappa}\right)
$$

as $\varepsilon \searrow 0$. The condition $\varkappa \leq l-1$ is only introduced in order to avoid more technicalities while introducing the step weighed space $V_{\varkappa, \beta}^{l-1}(\Omega ; \varepsilon)$ (compare [10]). Following the proofs we find that in the case $f_{4}=0$ this condition may be omitted, and we require $l \geq 1$ instead.

Let us point out some special combinations of weight indices and derivatives. If $\gamma=\varkappa$, then $\tilde{u}^{\varepsilon}=u^{\varepsilon}, \tilde{u}^{0}=u^{0}$, (no asymptotic is separated), this is the case of finite Dirichlet integral for the velocity parts. Suppose $f_{4}=0$, then for $l=\gamma=\varkappa=1$ we obtain from (7.8)

$$
\begin{array}{r}
\left\|r^{-1}\left(v^{0}-v^{\varepsilon}\right) ; L^{2}(\Omega)\right\|+\left\|\nabla\left(v^{0}-v^{\varepsilon}\right) ; L^{2}(\Omega)\right\|+\left\|p^{0}-p^{\varepsilon} ; L^{2}(\Omega)\right\| \\
\leq C \varepsilon\left(\left\|r f^{\prime} ; L^{2}(\Omega)+\right\| g^{\prime} ; H^{3 / 2}(\partial \Omega) \|\right) .
\end{array}
$$

For simplicity let also $g^{\prime}=0$. Then the same estimate applied to $l=2, \gamma=\varkappa$, leads to

$$
\begin{aligned}
\left\|r^{-1}\left(v^{0}-v^{\varepsilon}\right) ; L^{2}(\Omega)\right\|+ & \left\|\nabla\left(v^{0}-v^{\varepsilon}\right) ; L^{2}(\Omega)\right\|+\varepsilon\left\|\nabla^{2}\left(v^{0}-v^{\varepsilon}\right) ; L^{2}(\Omega)\right\| \\
& +\left\|p^{0}-p^{\varepsilon} ; L^{2}(\Omega)\right\|+\varepsilon\left\|\nabla\left(p^{0}-p^{\varepsilon}\right) ; L^{2}(\Omega)\right\| \\
\leq & C \varepsilon^{\varkappa}\left(\left\|f^{\prime} ; V_{\varkappa}^{\varkappa-1}(\Omega)\right\|+\varepsilon^{2-\varkappa}\left\|\nabla f^{\prime} ; V_{\varkappa}^{0}(\Omega)\right\|\right) .
\end{aligned}
$$

For $\varkappa \in(1,3 / 2)$ it follows that

$$
\begin{aligned}
& \left\|r^{-1}\left(v^{0}-v^{\varepsilon}\right) ; L^{2}(\Omega)\right\|+\left\|\nabla\left(v^{0}-v^{\varepsilon}\right) ; H^{1}(\Omega)\right\|+\left\|p^{0}-p^{\varepsilon} ; H^{1}(\Omega)\right\| \\
& \leq C\left(\varepsilon^{\varkappa-1}\left\|f^{\prime} ; V_{\varkappa}^{\varkappa-1}(\Omega)\right\|+\varepsilon\left\|\nabla f^{\prime} ; V_{\varkappa}^{0}(\Omega)\right\|\right)=O\left(\varepsilon^{\varkappa-1}\right)
\end{aligned}
$$

as $\varepsilon \searrow 0$. 


\section{References}

[1] W. Borchers and H. Sohr, On the equation $\operatorname{rot} v=g$ and $\Delta u=f$ with zero boundary conditions, Hokkaido Math. J. 19 (1993), 67-87.

[2] F. Brezzi and J. Pitkäranta, On the stabilization of finite element approximations of the Stokes equations, in: W. Hackbush (ed.), Efficient Solutions of Elliptic Systems, Notes on Numerical Fluid Mechanics 10, Vieweg, Braunschweig, 1984, 11-19.

[3] L. Cattabriga, Su un problema al contorno relativo al sistema di equazioni di Stokes. Rend. Sem. Mat. Univ. Padova 31 (1964), 308-340.

[4] M. Franta, J. Malek, and K. R. Rajagopal, On steady flows of fluids with pressure and shear dependent viscosities, Proc. R. Soc. Lond. Ser. A Math. Phys. Eng. Sci. 2055 (2005), 651-670.

[5] C. B. Morrey Jr., Multiple Integrals in the Calculus of Variations, Springer, Berlin, 1966.

[6] V. A. Kondratiev, Boundary value problems for elliptic equations in domains with conical or angular points, Trans. Moscow Math. Soc. 16 (1967), 227-313.

[7] J.-L. Lions and E. Magenes, Non-Homogeneous Boundary Value Problems and Applications, Vol. I, Springer, Berlin, 1972.

[8] S. A. Nazarov, Vishik-Lyusternik method for elliptic boundary-value problems in regions with conical points. 1. The problem in a cone, Siberian Math. J., 22 (1982), 594-611.

[9] S. A. Nazarov and B. A. Plamenevskii, Elliptic Problems in Domains with Piecewise Smooth Boundaries, de Gruyter, Berlin, 1994.

[10] S. A. Nazarov and M. Specovius-Neugebauer, Optimal results for the Brezzi-Pitkäranta approximation of viscous flow problems, Differential and Integral Equations 17 (2005), 1359-1394.

[11] A. Prohl, Projection and Quasi-Compressibility Methods for Solving the Incompressible Navier-Stokes Equations, Teubner, Stuttgart, 1997.

[12] H. Sohr, The Navier-Stokes Equations. An Elementary Functional Analytic Approach, Birkhäuser, Basel, 2001.

[13] H. Sohr and M. Specovius-Neugebauer, The Stokes problem for exterior domains in homogeneous Sobolev spaces, in: Theory of the Navier-Stokes equations, Ser. Adv. Math. Appl. Sci. 47, World Sci. Publ., River Edge, NJ, 1998, 185-205.

[14] M. Specovius-Neugebauer, Weak solution of the Stokes problem in weighted Sobolev spaces, Acta Appl. Math. 37 (1994), 195-203.

[15] M. Specovius-Neugebauer, Exterior Stokes problems and decay at infinity, Math. Meth. in the Appl. Sci. 8 (1986), 351-367.

[16] M. Specovius-Neugebauer, The Helmholtz decomposition of weighted $L^{r}$-spaces. Comm. Partial Differential Equations 15 (1990), 273-288.

[17] L. Tobiska and F. Lube, A modified streamline diffusion method for solving the stationary Navier-Stokes equation, Numer. Math. 59 (1991), 13-29.

[18] Ya. A. Routberg, Elliptic Boundary Value Problems in the Spaces of Distributions, Kluwer, Dordrecht, 1996. 